\title{
Development of a numerical model for fluid-structure interaction analysis of flow through and around an aquaculture net cage
}

\author{
Chen, Hao; Christensen, Erik Damgaard
}

Published in:

Ocean Engineering

Link to article, DOI:

10.1016/j.oceaneng.2017.07.033

Publication date:

2017

Document Version

Peer reviewed version

Link back to DTU Orbit

Citation (APA):

Chen, H., \& Christensen, E. D. (2017). Development of a numerical model for fluid-structure interaction analysis of flow through and around an aquaculture net cage. Ocean Engineering, 142, 597-615.

https://doi.org/10.1016/j.oceaneng.2017.07.033

\section{General rights}

Copyright and moral rights for the publications made accessible in the public portal are retained by the authors and/or other copyright owners and it is a condition of accessing publications that users recognise and abide by the legal requirements associated with these rights.

- Users may download and print one copy of any publication from the public portal for the purpose of private study or research.

- You may not further distribute the material or use it for any profit-making activity or commercial gain

- You may freely distribute the URL identifying the publication in the public portal 


\title{
Development of a numerical model for fluid-structure interaction analysis of flow through and around an aquaculture net cage
}

\author{
Hao Chen ${ }^{\mathrm{a}, *}$, Erik Damgaard Christensen ${ }^{\mathrm{a}}$ \\ ${ }^{a}$ Section of Fluid Mechanics, Coastal and Maritime Engineering, Department of Mechanical Engineering, Technical \\ University of Denmark, DK-2800 Kgs. Lyngby, Denmark
}

\begin{abstract}
In the present work, we developed a numerical model for fluid-structure interaction analysis of flow through and around an aquaculture net cage. The numerical model is based on the coupling between the porous media model and the lumped mass structural model. A novel interface was implemented to ensure efficient data exchange and element mapping between the fluid and structural solver via random-access memory. The main idea is to apply a static mesh in the fluid model, in case that large deformation of the net structure reduces the quality of the mesh. Then the geometry of the net cage was approximated by a set of dynamic porous zones, where the grid cells were updated at every iteration based on the transferred nodal positions from the structural model. A time stepping procedure was introduced, so the solver is applicable in both steady and unsteady conditions. In order to reduce the computational effort, sub-cycling was applied for the structural solver within each time step, based on the quasi-steady state assumption. The numerical model was validated against experiments in both steady and unsteady conditions. In general, the agreement is satisfactory.
\end{abstract}

Keywords: porous media model, lumped mass model, fluid-structure interaction analysis, aquaculture net cage, coupling scheme

\section{Introduction}

Aquaculture has been an important resource for food production in the world, and globally it is in a phase of steady expansion. As fresh water aquaculture has been increasingly constrained, space and water availability is driving aquaculture growth towards mariculture, from the bays and fjords with sheltered water to more exposed sites with large currents and waves. Therefore, the design for future offshore fish cages requires more accurate analysis and calculations.

Different numerical models have been proposed for analysis of hydrodynamic forces on the net structures. In general, they can be categorized into two approaches. One is the Morison type force model, where the net was modeled as individual twines. In many cases due to a large number of mesh in a net cage, the physical net cage was represented by an equivalent system of mesh with less twines. Morison equation was applied to calculate the forces on each twine, see e.g. Li et al. (2006), Moe et al. (2010), Tsukrov et al. (2002). Meanwhile, screen type force model has also been developed, where the net was divided into a number of screen elements. Each screen element is subjected to the same forces as on the twines and knots that are being represented. Due to a deflection of the flow through the screen, the resulted total force on a screen is not in the inflow direction, and it is usually decomposed into a drag and a lift component. Løland (1993) derived the drag and lift force coefficient based on fitting of the data from laboratory tests in Rudi et al. (1988). In Kristiansen and Faltinsen (2012), instead of using the curve fitting method, the drag and lift force coefficient were obtained as a function of solidity ratio, the angle between the panel normal and the

\footnotetext{
*Corresponding author, tel.: (45) 50302416; e-mail address: hchen@mek.dtu.dk; fax (45) 45251961.
} 
flow direction, and the drag force coefficient for the twines of the net in steady current, which is Reynolds number dependent. Kristiansen and Faltinsen (2015) demonstrates the application of this force model in waves. The elastic floating ring was also taken into account in the numerical model. Therefore, a complete rational model for a floating fish cage was set up.

Recently, the computational fluid dynamic (CFD) method combined with the porous media model was applied for simulating flow through such kind of porous structures in e.g. Patursson et al. (2010), Zhao et al. (2013b), Zhao et al. (2013a) etc. The net structure was modeled as a layer of porous media. An extra resistance was added in Navier-Stokes equations to represent the effects of the net structure on the fluid. The advantage of this method is that it is not necessary to model the detailed geometry of the net structure, which keeps the computational time on a reasonable level. Furthermore, both the hydrodynamic force on the net cage and the flow field through and around the net cage could be simulated in the numerical model. From the validation in the above-mentioned works, the numerical results agree well with the laboratory tests.

However, in reality, net structures are quite deformable under current and waves. The deformed structure will in turn affect the flow field. So, this is a typical fluid-structure interaction (FSI) problem. One needs to solve the governing equations in both the fluid and solid domain. In general, the numerical procedure to solve FSI problems could be broadly classified into two approaches: monolithic approach and partitioned approach (Hou et al., 2012). The monolithic approach treats the fluid and solid problems in the same mathematical framework. Hereby a single system of equations is formed finally for the entire problem in both the fluid and solid domain, which is solved at each time step. This indicates that the coupling is on a matrix level, and is fundamentally implicit, see e.g. Hübner et al. (2004), Le Tallec and Mouro (2001) and Michler et al. (2004) etc. This approach can potentially achieve better stability and accuracy, but it requires substantially much more resources and expertise to develop and maintain such a specialized code (Hou et al. 2012). Hereby instead of developing a monolithic solver from scratch, we decided to apply the partitioned approach. This means that the fluid and structural problem are solved separately with their respective numerical solver and mesh discretization. In the present work, the available CFD solver combined with a porous media model in the open source toolbox OpenFOAM is utilized as the fluid solver, while a custom lumped mass solver for the net cage is implemented as the structural solver.

Comparing with a conventional FSI solver, the coupling method between these two solvers is different for this particular problem, owing to its distinct characteristics. Usually a conventional FSI problem is a surface coupling problem, where the solid domain and the fluid domain are connected but not overlapped. There exists a clear interface which separates the fluid and solid domain, namely the boundary of the solid body. The interfacial conditions are explicit where the kinematic and dynamic boundary condition need to be fulfilled on the interface. However, for this problem, the geometry of the solid body (namely the net structure) is not resolved in the fluid solver. Instead, its effects are taken into account in a fictitious manner via an extra forcing term, i.e. the resistance in the Navier-Stokes equations. The forcing term is only active in the porous zone occupied by the net structure, and it is switched off anywhere else. Therefore, in the numerical model, the interface to separate the fluid and solid domain is not exactly reproduced, and the fluid is continuous throughout the whole computational domain. The deformation of the net structure is not represented by deformation of the interface, but motion of the resistance in the porous zones.

In principle, motion of the resistance could be realized in two ways in the numerical model. One is via motion of the internal subset of the mesh which represents the porous zones. This requires applying deforming mesh techniques. The internal subset of the mesh should conform with the deformation of the net structure, while the complementary part of the mesh is deformed based on the motion of the internal subset of the mesh. The benefit of this method is that the net deformation can be described accurately, since the motion of the internal subset of the mesh can in general match the deformation of the net very well. However, by using this method, the computational mesh may be significantly distorted, considering the negligible bending and compression stiffness of the netting materials. Therefore, we consider the second method, where a static mesh is applied. The instantaneous deformation of the net structure is represented by updating the grid cells in the porous zones, based on the solution from the structural solver. Conceptually this is similar to the immersed boundary method (Mittal and Iaccarino, 2005) for moving objects. The key point of this method is to find a fast and reliable algorithm for searching the grid cells that belong to the 
(a)

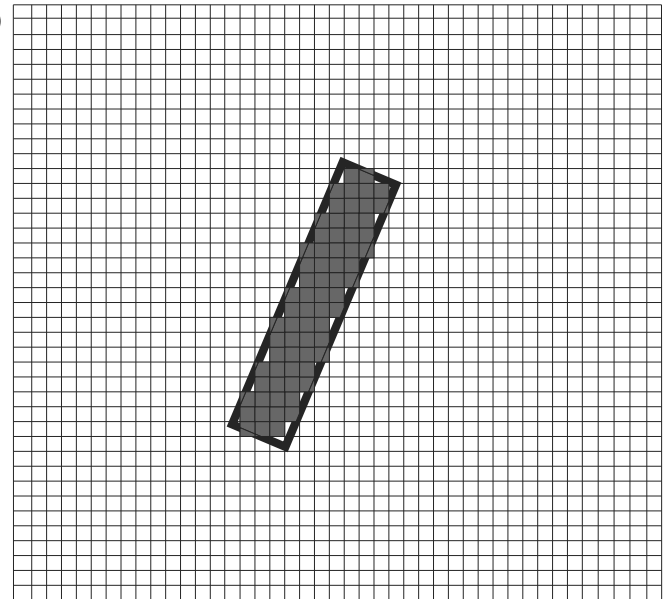

( b )

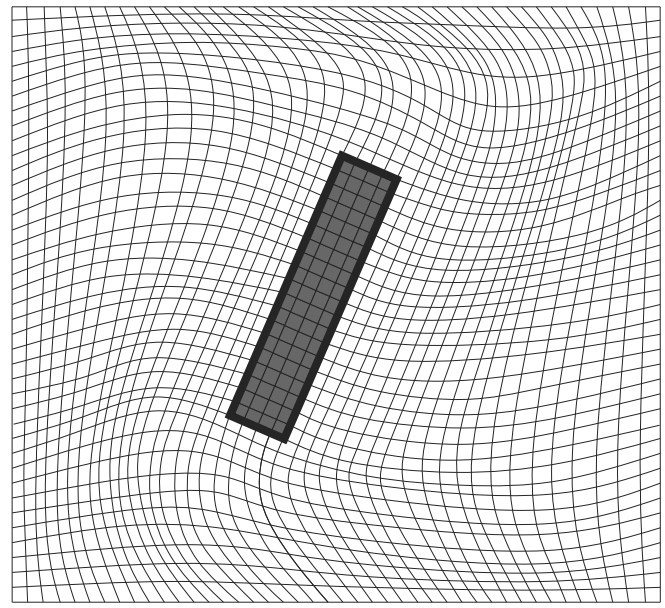

Figure 1: Illustration of the proposed two methods to represent a rotating porous zone. The bounding rectangular box with bold black lines stands for the boundary of the rotating porous zone, while the dark part stands for the actual porous zone using two methods. (a) Representation of the rotating porous zone using a static mesh with updated grid cells in the porous zone. (b) Representation of the rotating porous zone using the deforming mesh technique.

updated porous zones at each time step. Figure 1 gives an example on how these two methods are applied to represent a rotating porous zone. It is observed that by using the deforming mesh technique, the geometry of the rotating porous zone is accurately reproduced. However, this leads to a distortion in the complementary part of the mesh. It should be mentioned that Fig. 1(b) is only a graphic illustration on the deforming mesh technique, rather than any exact solution of a specific deforming mesh method. Actually, there exists plenty of methods, but essentially the nature of which is to solve different equations to relocate the grid cells for the updated moving boundary at every time step. Depending on the method that is applied, the distortion of the computational mesh can be either intensified or attenuated. But in general, for large amplitude of motion, especially for rotation motion, the distortion is significant irrespective of the method that is applied. However, in Fig. 1(a) with a static mesh, this issue is resolved. But we notice that the real boundary of the porous zone has zigzag fashion. Hereby a relatively fine mesh is required in the vicinity of the moving porous zones.

To the authors' knowledge, among those previous works on CFD simulation of flow though and around net structures, few considered the effects of net deformation, and they all applied the second method with static mesh. In Devilliers et al. (2016), a special solver was implemented for FSI analysis of current flow through net structures. The fluid solver solved pseudo-compressible Navier-Stokes equations, and the structure code DynamiT was applied to predict the net deformation, which approximated the net as a set of rigid bars. The resistance source term was estimated by Landweber-Ritchmeyer mechanic hypothesis, and the coupling was via output files. Advanced adaptive mesh refinement technique was applied to increase the resolution of the mesh in the net area. In Bi et al. (2014a) and Bi et al. (2014b), attempts have also been made to couple the porous media model with a lumped mass structural model. The coupling was based on the concept of "iteration". Under each iteration, steady state condition was assumed. The numerical model reached convergence very fast, usually under $1-3$ iterations. In the present paper, we will further improve the solver presented in Bi et al. (2014a) and Bi et al. (2014b). The main idea behind it is to achieve the FSI analysis in both steady and unsteady conditions. Hereby the linear and nonlinear waves can be properly generated in the numerical model.

The present paper is organized as follows. The fluid and structural solver that are applied in the numerical model are described in Section 2, Then Section 3 gives a thorough description on the coupling scheme between these two solvers. In Section 4 numerical study is carried out using the present model for three sets of cases, followed by a summary and conclusion in Section 5 . 


\section{Description of the fluid and structural solvers}

This is a typical hydro-elastic problem where the governing equations need to be solved in both fluid and solid domain. In the present work, we adopted an existing solver in OpenFOAM as the fluid solver, and implemented a lumped mass solver for the structural deformation of the net. Below in this section, these two solvers are described in detail.

\subsection{CFD solver}

The net structure in the fluid domain was represented by a sheet of porous media. This requires that the fluid solver has the capability on analysis of flow through porous structures. In Jensen et al. (2014) the governing equations on porous media flow were revised. The relevant library in OpenFOAM was reimplemented based on the new formulation, and it was released as open source together with the waves2Foam toolbox developed in Jacobsen et al. (2012). In the present work, this library was applied for both singlephase and two-phase flow solver in OpenFOAM. Below a brief description is given on it.

\subsubsection{Governing equations}

The governing equations for the CFD solver are the volume averaged Reynolds averaged Navier-Stokes (VARANS) equations:

$$
\begin{gathered}
\nabla \cdot\langle\overline{\boldsymbol{u}}\rangle=0 \\
\left(1+C_{m}\right) \frac{1}{n} \frac{\partial \rho\langle\overline{\boldsymbol{u}}\rangle}{\partial t}+\frac{1}{n} \nabla \cdot \frac{\rho}{n}\langle\overline{\boldsymbol{u}}\rangle\langle\overline{\boldsymbol{u}}\rangle^{T}=-\nabla\langle\bar{p}\rangle^{f}-\boldsymbol{g} \cdot \boldsymbol{x} \nabla \rho+\frac{1}{n} \nabla \cdot \mu \nabla\langle\overline{\boldsymbol{u}}\rangle+\boldsymbol{S}
\end{gathered}
$$

where $\boldsymbol{x}$ is the global Cartesian coordinate system, $\langle\overline{\boldsymbol{u}}\rangle$ is the volume averaged ensemble averaged velocity field, $\rho$ is the density of the fluid, $\mu$ is the dynamic viscosity, $n$ is the porosity of the net, $\boldsymbol{g}$ is the gravitation, $p$ is the excess pressure defined as

$$
p=P-\rho \boldsymbol{g} \cdot \boldsymbol{x}
$$

where $P$ is the total pressure. Therefore, $\langle\bar{p}\rangle^{f}$ is the intrinsic volume averaged ensemble averaged excess pressure, where \langle\rangle$^{f}$ is the intrinsic volume average. It relates to the volume average in the form of

$$
\langle p\rangle=n\langle p\rangle^{f}
$$

Here intrinsic volume average is preferred for the pressure since it corresponds to the actual measured pore pressure, as revealed in Jensen et al. (2014). $S$ is the resistance force due to the presence of the porous media, and $C_{m}$ is the added mass coefficient, which was calculated as:

$$
C_{m}=\gamma_{p} \frac{1-n}{n}
$$

where $\gamma_{p}$ is an empirical coefficient, and takes the value of 0.34 . The resistance force used in Eq. (22) was expressed as:

$$
\boldsymbol{S}=-\frac{1}{2} \rho C\left|\langle\overline{\boldsymbol{u}}\rangle-\boldsymbol{u}_{\boldsymbol{n}}\right|\left(\langle\overline{\boldsymbol{u}}\rangle-\boldsymbol{u}_{\boldsymbol{n}}\right)
$$

where $\boldsymbol{u}_{n}$ is the velocity of the net structures. Eq. (6) uses relative velocity for the resistance. This is necessary when there exists relative motion between the fluid and net structure. $C$ is the quadratic porous resistance coefficient matrix. In its local coordinate system it was given as:

$$
C=\left[\begin{array}{ccc}
C_{1} & 0 & 0 \\
0 & C_{2} & 0 \\
0 & 0 & C_{3}
\end{array}\right]
$$

If the local coordinate system is not aligned with the global coordinate system, transformation of the coefficient matrix is needed. Due to deformation of the net, the transformation matrix is not constant. It will be re-calculated at every time step. 
If free surface effect needs to be considered (e.g. waves are modeled in the numerical simulation), an additional equation needs to be solved based on the available Weller-VOF scheme to capture the interface:

$$
\frac{\partial \alpha}{\partial t}+\frac{1}{n} \nabla \cdot\langle\overline{\boldsymbol{u}}\rangle \alpha+\frac{1}{n} \cdot\left\langle\overline{\boldsymbol{u}}_{r}\right\rangle \alpha(1-\alpha)=0
$$

where $\alpha$ is the volume fraction field, $\boldsymbol{u}_{r}$ is the relative velocity (see Berberović et al. (2009) for details), which aids to maintain a sharp interface. The correction of $1 / n$ in Eq. (8) ensures that only the pore volume can be filled with water (see e.g. Jensen et al. (2014) and Jacobsen et al. (2015)).

This set of VARANS equations have already been successfully applied in our previous work in Chen and Christensen (2016) for current and wave interaction with fixed net structures. Specifically, we discussed on the formulation of resistance force $S$, the role of porosity $n$ in Eq. 22 and the added mass force etc. Readers are referred to that paper for relevant information.

\subsubsection{The solution algorithm}

The PIMPLE algorithm was employed to deal with the velocity-pressure coupling in Navier-Stokes equations. It essentially merges the SIMPLE (Semi-Implicit Method for Pressure-Linked Equations, see Patankar and Spalding (1972)) and PISO (Pressure Implicit with Splitting of Operators, see Issa (1986)) algorithm. The main characteristic of this algorithm is to introduce PIMPLE iterations within each time step. The main structure of PIMPLE iteration was inherited from the original PISO algorithm, but it allows under-relaxation of equations in the end of each iteration. With sufficient PIMPLE iterations in each time step, convergence of equations can be ensured at each time step.

Regarding the simulation with single-phase flow, PIMPLE algorithm allows large time steps, which relaxes the constraint of Courant number and maintains the stability of the solver. However, for two-phase flow, a special designed solver called multi-dimensional limiter for explicit solution (MULES) solver was applied to solve Eq. (8). This solver effectively guarantees the boundness of the volume fraction field $\alpha$, but it comes at a cost that MULES solver is explicit. Therefore, the time step should be limited by Courant number strictly, see Section 3.3 for detailed introduction on the selection of time step. An explicit method was applied for discretization of the resistance term, i.e. it was fully treated as a source term at the right-hand side of the discretized equation.

\subsection{Lumped mass structural solver}

The lumped mass solver presented in Lader and Fredheim (2006) and Lader et al. (2003) was employed as the structural solver. This solver was chosen because of its simple formulation, and it is relatively easy to implement within the framework of OpenFOAM toolbox.

\subsubsection{Overview}

The net structure was represented by a set of panel elements and nodes in the lumped mass model. For $2 \mathrm{D}$ cases, each element was associated with two nodes, while each node was restricted to move within the vertical plane. Furthermore, the panel elements were assumed not to be rotated around the vertical axis. Consequently, the element normal unit vector was restricted to be parallel with the vertical plane. For 3D case, each panel element was associated with four nodes located at the corners of the element, and each node was allowed for full 3D movement. Therefore, for the deformed nets, the four nodes associated with the same element were not necessarily in the same plane. Fig. 2 depicts the general configuration of the model for $2 \mathrm{D}$ and $3 \mathrm{D}$ cases. The mass and the forces acting on the elements were distributed uniformly into its associated nodes, and the equation of motion was evaluated at every node.

\subsubsection{Forces on the node}

The forces acting on each node include hydrodynamic force $F_{h}$, structural force $F_{s}$, gravity force $F_{g}$ and buoyancy force $F_{w} . F_{w}$ and $F_{g}$ were constant and completely determined by the net properties. $F_{h}$ was calculated based on the solution from the CFD model:

$$
\boldsymbol{F}_{h}=\int_{V_{P}} \frac{1}{2} \rho C\left|\langle\overline{\boldsymbol{u}}\rangle-\boldsymbol{u}_{\boldsymbol{n}}\right|\left(\langle\overline{\boldsymbol{u}}\rangle-\boldsymbol{u}_{\boldsymbol{n}}\right) \mathrm{d} V
$$




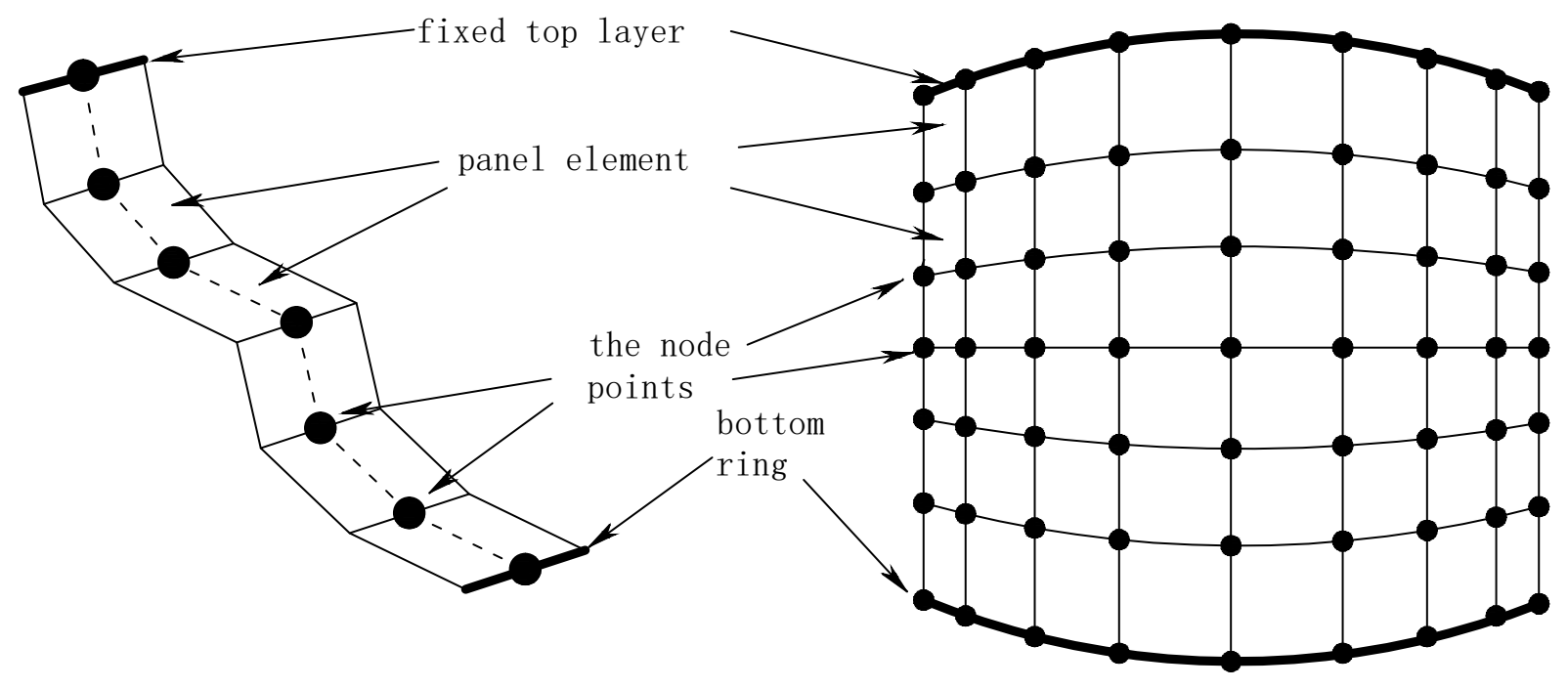

Figure 2: Illustration on the lumped mass structural model for modeling top fixed and bottom weighted net cages. The net is represented by a set of nodes and panel elements. Left: $2 \mathrm{D}$ case, where the nodes are placed in the intersection between the panel elements. Right: 3D case, where the nodes are placed in the corner of the panel elements.

where $V_{p}$ is the volume of the porous zones. This is simply the integration of the reaction force of the porous resistance over the porous zones. Regarding the structural force, the elastic property of the net structure in the model was represented by a nonlinear spring, and the relationship between the force and elongation $\epsilon$ was expressed as:

$$
F_{s}= \begin{cases}C_{1} \epsilon+C_{2} \epsilon^{2} & \epsilon>0 \\ 0 & \epsilon \leq 0\end{cases}
$$

So, the netting material was assumed to only have tension stiffness, where the compression stiffness was neglected. $C_{1}$ and $C_{2}$ are constants that define the elasticity of the element. $\epsilon$ is the global elongation, defined as $\left(l-l_{0}\right) / l_{0}$, where $l_{0}$ is the undeformed side length and $l$ is the deformed side length of the element. In the present work $C_{1}$ and $C_{2}$ were taken from Lader and Fredheim $(2006)$ as $C_{1}=1160 \mathrm{~N}$ and $C_{2}=37300 \mathrm{~N}$.

\subsubsection{Equation of motion}

The forces acting on each element were uniformly distributed into all the nodes of the element, then the equation of motion was evaluated at every node:

$$
\boldsymbol{F}_{s}+\boldsymbol{F}_{h}+\boldsymbol{F}_{g}+\boldsymbol{F}_{w}=m_{n} \boldsymbol{a}_{\boldsymbol{n}}
$$

where $m_{n}$ is the structural mass that is distributed to each node, and $\boldsymbol{a}_{n}$ is the acceleration for each node.

Fig. 3 gives an example on the forces acting on each node in 2D case. Each element was connected by two nodes. Therefore, the hydrodynamic force acting on the element was distributed into these two nodes. Meanwhile, the structural force on each node was calculated based on the position of the node itself and its neighbors. The motion equation was then evaluated at each node to obtain its acceleration.

\subsubsection{Time integration}

The displacement and velocity of each node were obtained from time integration of its acceleration:

$$
\boldsymbol{u}_{n}=\int \boldsymbol{a}_{n} \mathrm{~d} t \quad \boldsymbol{x}_{n}=\int \boldsymbol{u}_{n} \mathrm{~d} t
$$



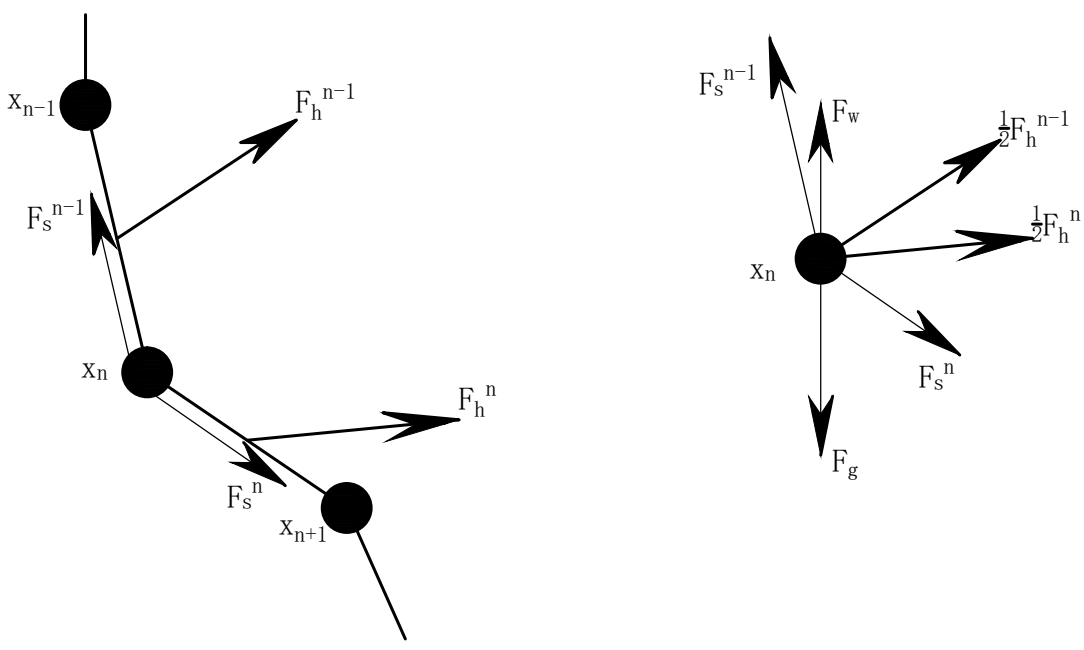

Figure 3: Left: hydrodynamic and structural forces acting on the panel elements and the nodes in $2 \mathrm{D}$ case. $x_{n}$ is the node located at the intersection between the panel element $n-1$ and $n . x_{n-1}$ and $x_{n+1}$ are its neighboring nodes. $F_{h}^{n-1}$ and $F_{h}^{n}$ are the hydrodynamic forces acting on the panel element $n-1$ and $n . F_{s}^{n-1}$ and $F_{s}^{n}$ are the structural forces on the node $x_{n}$ due to its neighboring node $x_{n-1}$ and $x_{n+1}$. Right: the forces that are distributed on the node $x_{n}$.

An explicit fourth order Runge-Kutta method provided in OpenFOAM library was applied for the integration. The problem has quite stiff characteristics and the time step needs to be very small, in particular at the beginning of the simulation. Therefore, a quasi-steady state approximation was applied for the coupling of the two solvers to accelerate the computation. This will be introduced in detail in Section 3 .

\section{Coupling between two solvers}

The main contribution of this work is the coupling scheme between two solvers. The use of open source software OpenFOAM as the fluid solver and the author-implemented lumped mass structural solver enable both solvers to be combined into a single executable. Both solvers were written in an object-oriented manner. Therefore, the coupling between them was facilitated. Data exchange between the solvers was through random-access memory rather than output files to increase the computational speed. Below, details will be given on this coupling scheme.

\subsection{Overview}

The coupling scheme is an extension of the original PIMPLE algorithm. The idea is to couple the structural solver inside each PIMPLE iteration within each time step. The flow chart of the algorithm for the solver is presented in Fig. 4. At the beginning of the simulation, the physical parameters of the net structure were read by the solver, and the initial configuration of the net structure was calculated. Corresponding to this initial configuration, the fluid solver created the porous zones, while the structural solver initialized the position vector field for the computation.

When the simulation started, at each PIMPLE iteration, the $\alpha$ field was obtained from the MULES solver first if waves were involved in the simulation. Based on the $\alpha$ field, the density and viscosity field were updated. Then the porous resistance coefficients were transformed into the global coordinate system based on the position of the porous zones from last iteration. In addition, the porous resistance was calculated and added to the momentum equation. To begin with the PISO algorithm, the momentum predictor produced an intermediate velocity field, where the pressure field in the equation was obtained from last iteration. This velocity field was not divergence-free, hereby it was followed by three pressure correctors to correct 
the velocity field. A divergence-free velocity field was produced after the correction, and it was used to calculate the hydrodynamic forces on the structural panel elements. Based on the received hydrodynamic forces, the structural solver evaluated the position of each node, from which the porous zones were updated. This formed a closed PIMPLE iteration. At every time step three PIMPLE iterations were performed to get rid of the stability issues.

In the numerical model, the interaction effects between the flow and the net were reflected as below:

- The geometry of the net was updated at every PIMPLE iteration according to the solution from the structural solver.

- The transformation matrix for the porous resistance coefficients was updated at every PIMPLE iteration based on the instantaneous orientation of the porous zones.

- The porous resistance force was calculated based on the instantaneous relative velocity between the flow and the net, and the instantaneous porous coefficient matrix in the global coordinate system.

\subsection{Data exchange and mapping of elements}

The basic idea for element mapping in the present work is to adopt a one to one mapping method between the elements in the structural and fluid model. Therefore, in principle interpolation is not needed. However, in practice, the structural elements are significantly larger than the mesh cells in the fluid model. Hereby we decided to use the structural element as the fundamental element for date exchange. The corresponding element in the fluid model is the porous zone, i.e. in the fluid model, the geometry of the net was approximated by a set of dynamic porous zones.

The data that need to be exchanged at run time are the porous forces and the nodal positions of the structural elements. The nodal positions were transferred from the structural model to the fluid model. Since a static mesh was used in the simulation, one needs to update the porous zones at every iteration based on the transferred nodal positions, which was not straightforward. It was resolved by first clearing the mesh cells in the porous zones, then looping through all the cells in the mesh to identify the cells that fulfill the conditions. The conditions are typically a set of mathematical inequalities derived from the nodal positions and the thickness of the porous zones. The detailed derivation is revealed in Appendix A.

\subsection{Quasi steady-state assumption and sub-cycling}

As mentioned above in Section 2.1.2, the PIMPLE algorithm allows large time steps for simulation of single-phase flow. However, as described in Section 2.1.2 for two-phase flow the MULES solver is fundamentally explicit. The time step is limited by Courant number, which relates the time step $\Delta t$ to the local cell velocity and the cell size $\Delta x$. For one dimensional case, it was expressed as:

$$
C o=\frac{\Delta t|\boldsymbol{u}|}{\Delta x}
$$

In principle, in order to ensure the stability of the numerical model, the maximum value of Courant number should be smaller than one throughout the whole domain. But we found that for wave making purpose, the Courant number should be controlled below 0.25 , to improve the accuracy of wave making in the numerical model. This constrains the time step in the order of $10^{-3} \mathrm{~s}$. However, the structural solver requires an even smaller time step, typically around $10^{-4} \sim 10^{-5} \mathrm{~s}$. This is due to the reason that the structural solver is also explicit, and the ordinary differential equations that we solved, i.e. Eq. 12 have quite stiff characteristics due to high structural eigen-frequency as shown in Lader et al. (2003).

In order to accelerate the computation, the time step for the whole solver was chosen based on the fluid solver, and quasi-steady state assumption was applied within the PIMPLE iteration. This means that during each iteration, the hydrodynamic forces output from the fluid solver were assumed to be constant, and the position of the net was evolved under the constant forces. A much smaller time step was applied for the evolution of the structural position, typically $1 / 100 \sim 1 / 1000$ of the time step for the global solver, depending on the simulated cases. Therefore, sub-cycling was applied within each PIMPLE iteration. Since the computational time for the fluid model was significantly more than the structural model, the computational speed was dramatically improved by application of this assumption. 

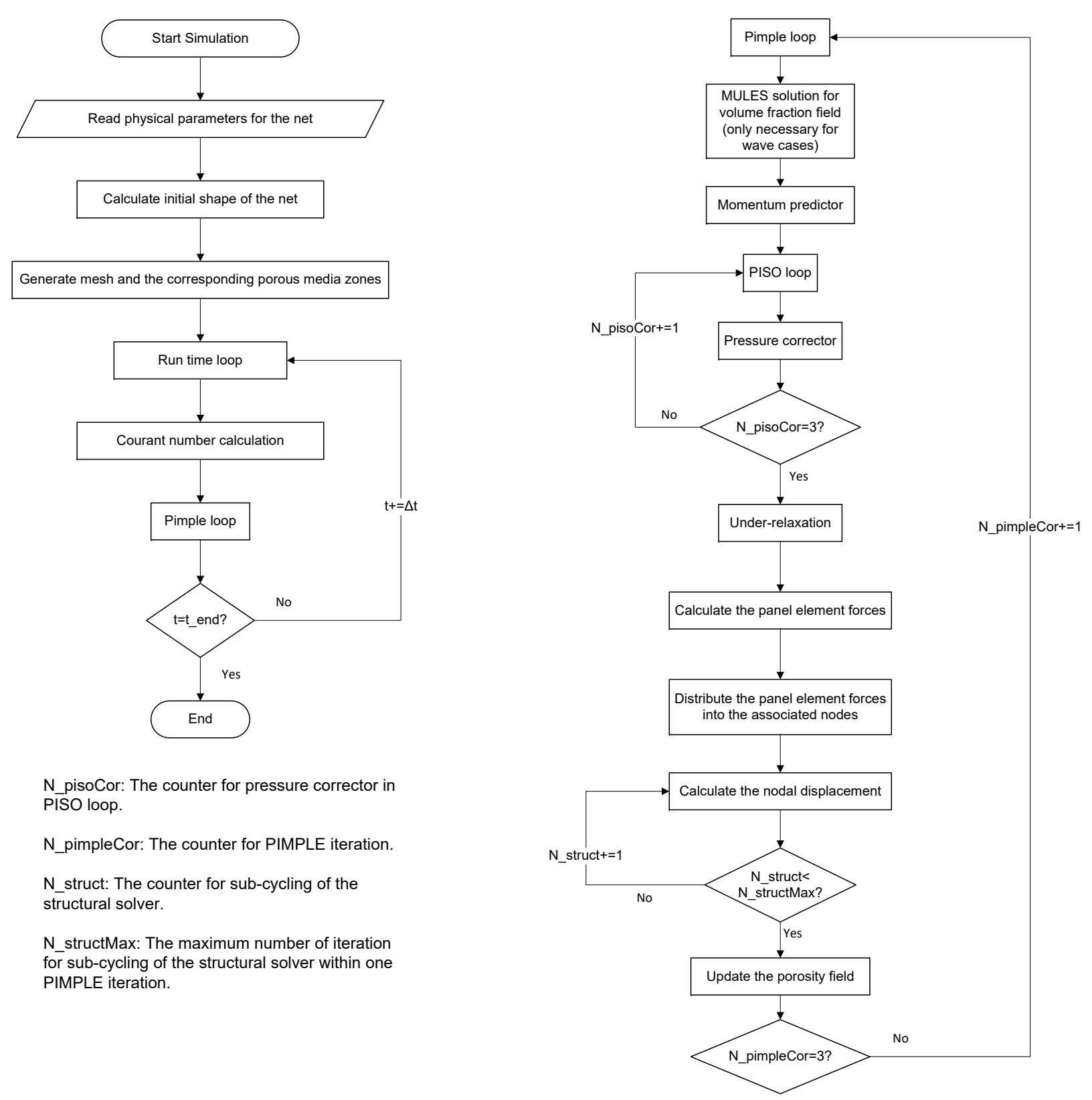

$\mathrm{N}$ pisoCor: The counter for pressure corrector in PISO loop.

N_pimpleCor: The counter for PIMPLE iteration.

N_struct: The counter for sub-cycling of the structural solver.

N_structMax: The maximum number of iteration for sub-cycling of the structural solver within one PIMPLE iteration.

Figure 4: The flow chart of the algorithm for the solver. 


\section{Numerical study and sensitivity analysis}

In this section, series of simulations are carried out using the numerical model described above. The purpose is to examine the performance of the numerical model under different net geometries and environmental conditions. First the numerical model is validated against experiments in Bi et al. (2014b) for plane net panel in steady current. Considering that the simulation for this case is not time consuming, we perform sensitivity analysis to investigate the influences of the parameters in the numerical model. Furthermore, the model is also validated against experiments in Zhao et al. (2008) and Bi et al. (2014a), for plane net panel in waves and circular net cages in steady current. In all the validation cases, the nets are fixed at the top layer, and at the bottom layer a sinker is attached. Under different flow velocities, the nets can deform freely, which in turn affects the flow field. Therefore, the flow field and the structural deformation are coupled strongly, and they are used to properly validate our numerical model.

\subsection{Plane net panel in steady current flow}

The first set of experiments was described in Bi et al. (2014b), which was performed in a wave-current flume at the State Key Laboratory of Coastal and Offshore Engineering, Dalian University of Technology, China. The flume had a dimension of $22 \mathrm{~m} \times 0.45 \mathrm{~m} \times 0.6 \mathrm{~m}$, and the depth of the water was set to $0.4 \mathrm{~m}$ in the tests. Steady current with the incoming velocity $U=0.226 \mathrm{~m} / \mathrm{s}$ was generated by a pump located at one end of the flume.

The net used in the experiments was a $0.3 \mathrm{~m} \times 0.3 \mathrm{~m}$ knotless polyethylene net with $15 \times 15$ meshes horizontally and vertically. The twine diameter $d_{w}=2.6 \mathrm{~mm}$, and the mesh bar length $\lambda=20 \mathrm{~mm}$. This gave the solidity ratio $S_{n}=0.26$. This net was positioned in the center of the flume and just below the free surface. A steel bar with density of $8610 \mathrm{~kg} / \mathrm{m}^{3}$ was mounted at the bottom of the net as a sinker system. It had the same length as the net panel, and the diameter of it was $6 \mathrm{~mm}$. The weight of the sinker was 73 $\mathrm{g}$ in air while in water it was $64.5 \mathrm{~g}$ by subtracting the buoyancy.

A 2D numerical model was set up based on the experiment. The single-phase flow solver was applied as the fluid solver, where free surface was not modeled. The sketch of the computational domain and the boundary condition is given in Fig. 5. The net was divided into 5 structural elements. The mass of each structural element was uniformly distributed into its two nodes, and the weight of the sinker was added to the lumped mass point at the bottom. In the fluid model, the thickness of the porous media zone $t_{0}$ was set to $20 \mathrm{~mm}$, and the porous resistance coefficients were calculated based on the formula proposed in Chen and Christensen (2016), where $C_{1}=27.9 \mathrm{~m}^{-1}$ and $C_{2}=C_{3}=9.0 \mathrm{~m}^{-1}$. The sinker was not modeled in the fluid model, but the forces acting on it were directly added to the corresponding lumped mass point in the structural model. This includes the weight of the sinker in water, where the buoyancy was subtracted, and the mean drag force, which was calculated based on Morison equation.

\subsubsection{Net deformation}

The deformation of the net panel is presented in Fig. 6. In general, the numerical model reproduced the deformation of the net reasonably, where the displacement at the bottom of the net was $0.19 \mathrm{~m}$ from both the numerical model and the experiment. However, it was observed that some of the porous cells were missing between the connecting structural elements, which have been encircled by the black circles as shown in Fig. 6. This is due to the imperfection of the algorithm to update the porous zones, and the application of the static mesh, which is naturally only possible to approximate the geometry of the deformed net. These missing cells will have side effects on the wake in the downstream, which will be discussed in Section 4.1.2.

\subsubsection{Flow field}

Physically, when flow passes through a net panel, a wake region will form behind it. Define the Reynolds number of the net panel $R e_{n}$ as

$$
R e_{n}=\frac{U d_{w}}{\nu\left(1-S_{n}\right)}
$$

where $\nu$ is the kinematic viscosity, and $1-S_{n}$ in the denominator accounts for local speed-up of the flow when passing through the net, as shown in Kristiansen and Faltinsen (2012). Then in this case the Reynolds 


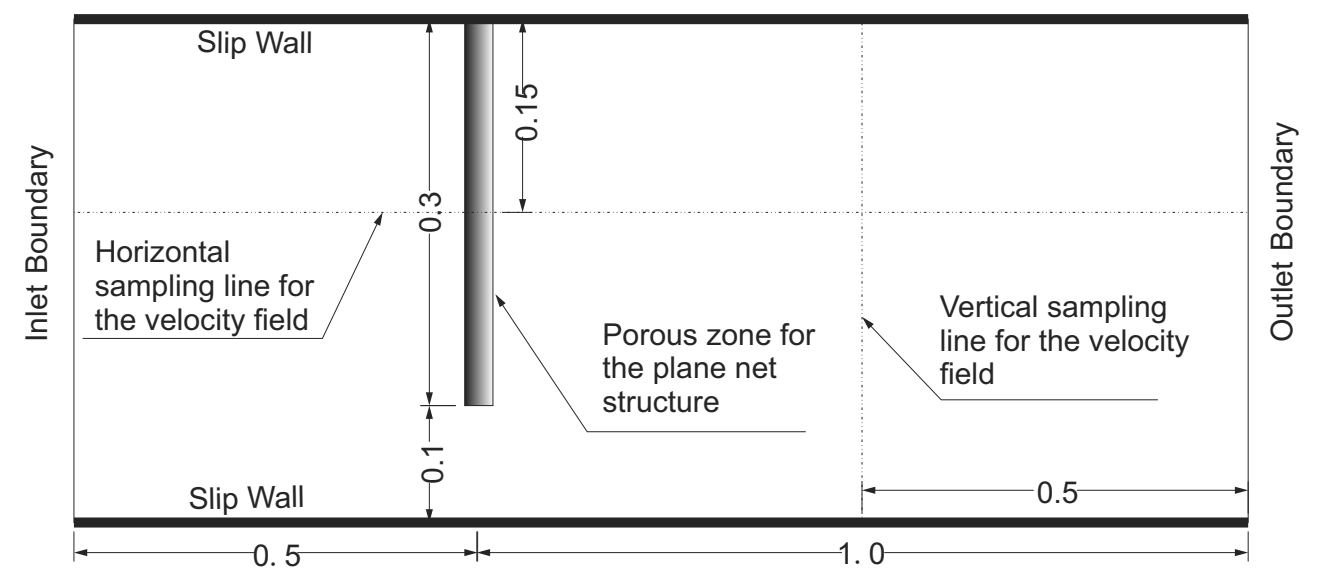

Figure 5: The computational domain and the boundary condition for simulation of plane net structure in steady current (unit: $\mathrm{m}$ ). The bottom and top boundary were modeled as slip walls, and the free surface was not modeled. The porous zone representing the plane net structure has a thickness of $20 \mathrm{~mm}$ and the sinker of the net was not modeled. The velocity field was sampled horizontally at $0.15 \mathrm{~m}$ below the top boundary and vertically 0.5 behind the plane net. The sampled velocity is given in Fig. 8

number is 794 . This indicates that the wake is completely turbulent, while the boundary layer remains laminar. The velocity in the wake is smaller than in the main flow. The loss of velocity in the wake occurs as a result of a loss of momentum owing to the drag of the net (Løland, 1993). In addition, due to the difference of the velocities in the wake and outside the wake below the net (namely the outer region), a transition zone will be formulated. The flow in the transition zone is free shear flow, which has boundary layer nature. In Bi et al. (2013), the velocity distribution has been measured across the wake to the outer region (see e.g. Fig. 7 in Bi et al. (2013)), which provides evidence on how the velocity is distributed in the transition zone. Below in this section, the flow field from the numerical simulation is examined, especially in the wake and the transition zone.

Fig. 7 presents the velocity field using laminar and $k-\epsilon$ turbulence model. By comparing with Fig. 7(b), two distinctions were observed in 7(a) for the case with laminar model. The first distinction is in the wake region. As mentioned in Section 4.1.1, the missing porous cells have effects on the wake velocity. Actually, the missing porous cells lead to slightly higher velocity in the wake behind it. In Fig. 7(a) it has been clearly marked by rectangle 1 , which is located at $y=-0.15 \mathrm{~m}$. The higher velocity was resulted by the missing cells encircled by the lower black circle in Fig. 6. However, in Fig. 7(b), the wake velocity was distributed more uniformly. The extra turbulent diffusion causes momentum exchanges in-between the layers with different velocities, therefore smooths the velocity profiles. This is further demonstrated quantitatively in Fig. 8 by sampling the velocity horizontally and vertically. The location of the sampling has been given in Fig. 5 , namely $0.15 \mathrm{~m}$ below the free surface and $0.5 \mathrm{~m}$ downstream behind the net panel. Fig. 8(a) presents the velocity distribution horizontally at $0.15 \mathrm{~m}$ below the free surface by using different turbulence models. It is noticed that the same reduced velocities were obtained by considering turbulent effects, and it is slightly higher than the wake velocity from the laminar model. Furthermore, spikes were observed for the wake velocity from the laminar model (the irregularities of the green curve). This is again due to the missing cells encircled by the lower black circle in Fig. 6. The engineering approach provided in Løland (1993) is also included in Fig. 8(a) for comparison. It is suggested that the reduced velocity in the wake can be predicted as $r U$, where $r$ is calculated as

$$
r=1-0.46 C_{d}
$$

where $C_{d}$ is the drag force coefficient of the net panel. Furthermore, the experimental data from Bi et al. (2014b) is also given in Fig. 8(a). It was observed that comparing with the experimental result, the wake velocity was overpredicted by the numerical models, but the overprediction was within $4 \%$. Meanwhile Løland formula underpredicted the wake velocity for about $7 \%$. 

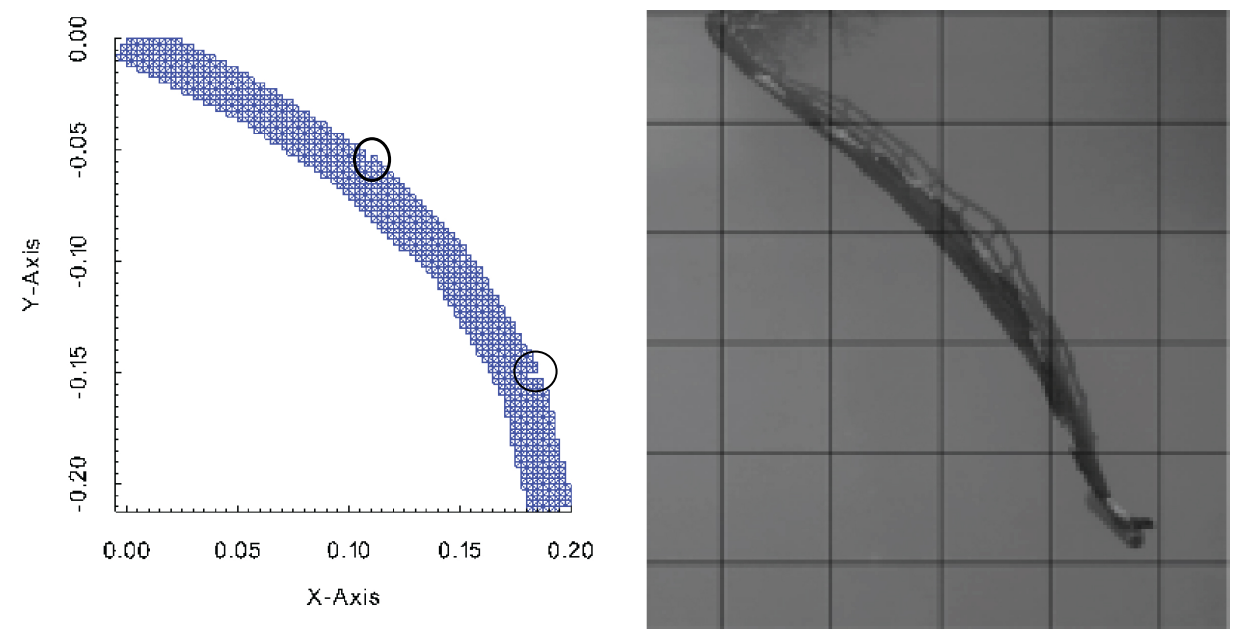

Figure 6: Comparison of the net deformation between the numerical simulation (left) and the experimental result (right). In the left figure the plane net structure is represented by the porous zones in the blue area. The coordinate system is given to quantify the deformation. The black circles denote the missing cells between the connecting structural elements. The right figure was reproduced from Fig. $19 \mathrm{in}$ Bi et al. (2014b), where each mesh has a length of $50 \mathrm{~mm}$.

In Fig. 8(b), the velocity distribution in vertical direction is presented. Spikes were also observed for the laminar model, which were induced by the missing porous cells over the porous zones. Furthermore, the velocity profile in the transition zone was analyzed. It was noticed that the laminar model produced a very sharp velocity gradient in the transition zone. However, with a turbulence model, the sharp velocity gradient was smeared out. Comparing with other turbulence models, realizable $k-\epsilon$ model gave a less diffusive result. In order to verify the result, the analytical solution based on laminar and turbulent mixing layer flow were also presented in Fig. 8(b). It was assumed that the wake velocity and the velocity in the outer region were uniformly distributed vertically. Therefore, the flow was simplified as two parallel streams with the mean velocities in the wake and the outer region, namely $U=0.247 \mathrm{~m} / \mathrm{s}$ in the outer region and $\lambda U=0.207 \mathrm{~m} / \mathrm{s}$ in the wake, where $\lambda$ denotes the ratio between the velocity in the wake and outer region $(0<\lambda<1)$. The derivation of the solution has been given in Appendix B. It was observed that the laminar mixing layer theory produced a similar result with the laminar model from the numerical simulation. However, the turbulent mixing layer flow produced a less diffusive result, which is close to the result from realizable $k-\epsilon$ model.

\subsubsection{Current load on the net panel}

We directly output the time series of the drag force from the numerical computation. It was found that an oscillation-free time series requires a small time step for both the fluid solver, i.e. the global time step and the structural solver. Fig. 9 depicts the time series of the drag force under different combination of Courant number, and the number of sub-cycling. It was observed that only case (e) was qualified for an oscillation-free time series. In addition, the amplitude of the oscillation depends on both Courant number and the number of sub-cycling. In Fig. 9(f), the mean drag force for each case is reported with the result from the laboratory test.

It should be noted that the oscillation of the load is resulted by the artifacts of the numerical method. Physically in the laboratory tests, oscillation of steady current load was also observed due to e.g. vortex shedding behind the net panel. However, in the present numerical model, the geometry of the net was not modeled. Therefore, the boundary layer around the twines and knots of the net panel was neither modeled, and the vortex shedding phenomenon cannot be captured. Meanwhile, since the Reynolds averaged NavierStokes equations were applied, the mean quantity over time should be output from the numerical model. However, recall that the current load was calculated based on Eq. (9), which was a function of both the fluid velocity and the velocity of the structural node. Therefore, it was affected by both the global time step and 
(a)

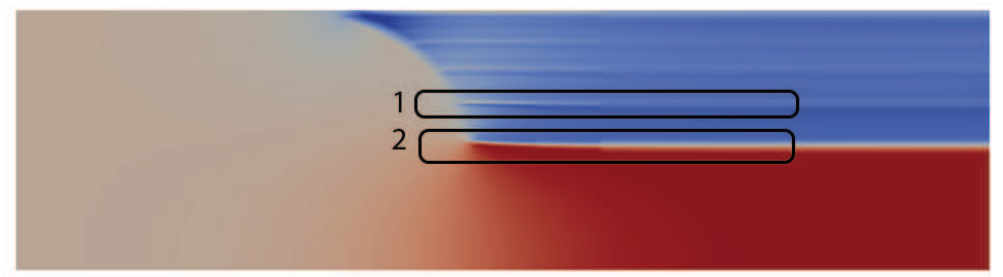

U

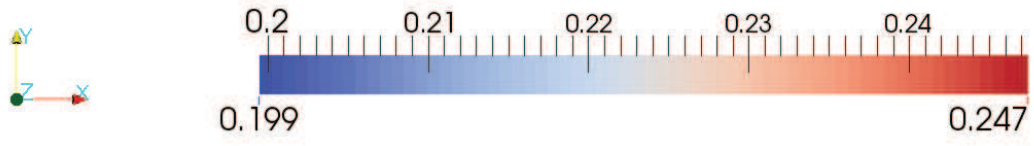

(b)

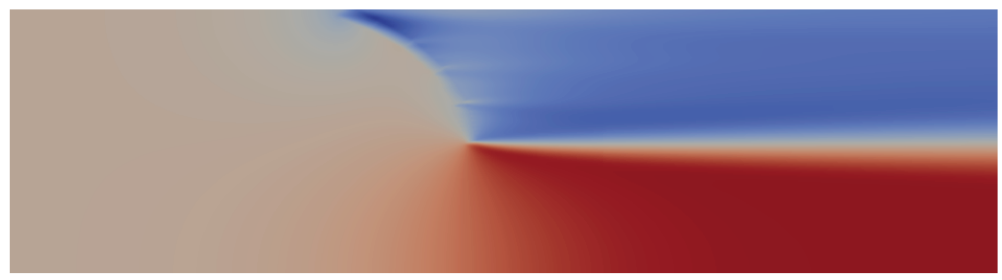

U

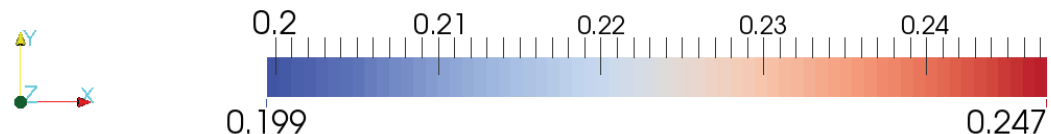

Figure 7: Snapshot of the velocity field for the deformed net panel in steady current flow. (a) The simulated flow field using laminar model. The rectangle 1 marks the oscillation of the velocities in the wake behind the net panel. The rectangle 2 marks the sharp velocity gradient in the transition zone from the wake to the outer region, namely the region below the net. (b) The simulated flow field using realizable $k-\epsilon$ model.
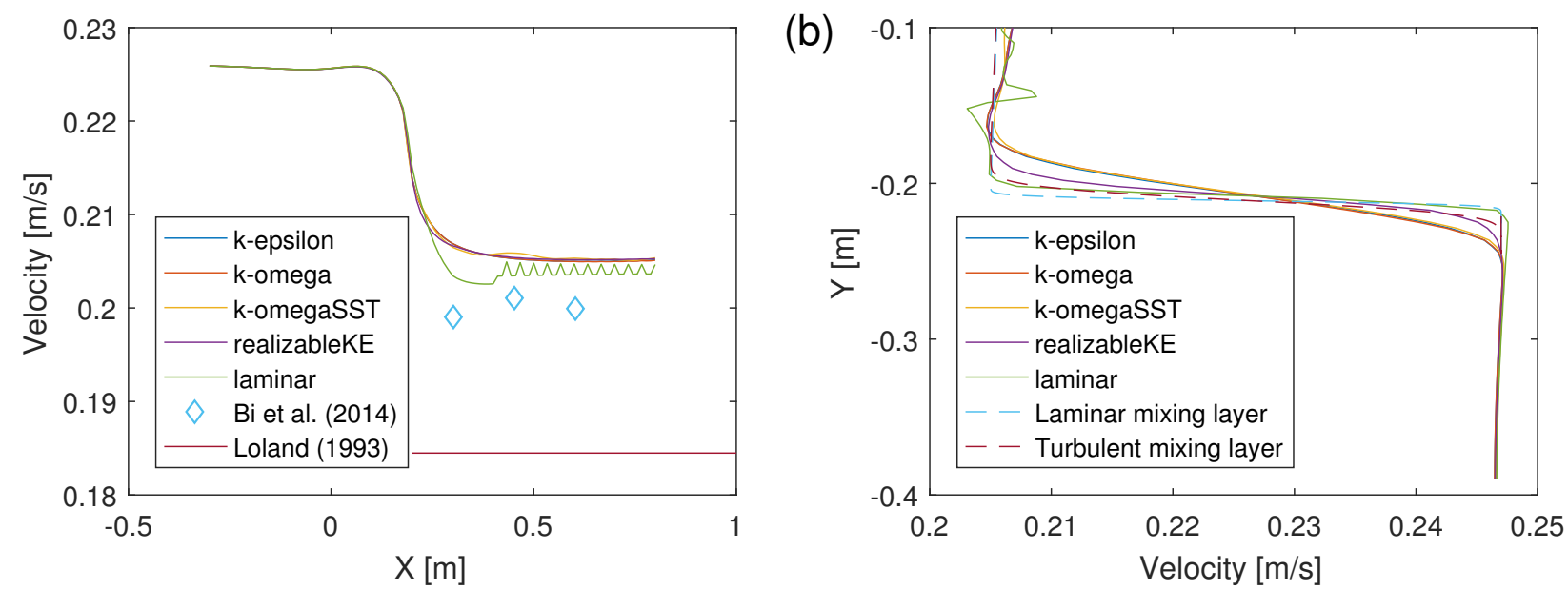

Figure 8: Horizontal and vertical sample of the velocity field from the numerical simulations with laminar model, $k-\epsilon$ model, $k-\omega$ model, $k-\omega$ SST model and realizable $k-\epsilon$ model. The location of the sampling is described in Fig. 5 (a) Horizontal sampling of the velocity field at $0.15 \mathrm{~m}$ below the free surface. The experimental data from Bi et al. (2014b) and the result based on the formula in Løland (1993) are also presented for the purpose of verification and validation. (b) Vertical sampling of the velocity field at $0.5 \mathrm{~m}$ behind the net panel. The results based on laminar and turbulent mixing layer flow are also presented for verification purpose. 
(a)

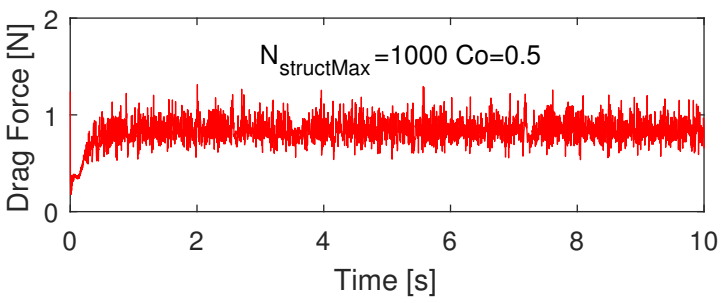

(c)

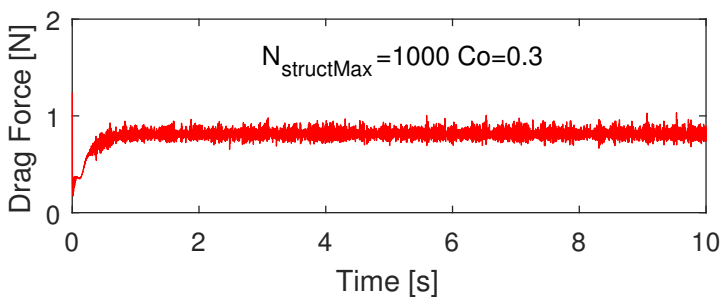

(b)

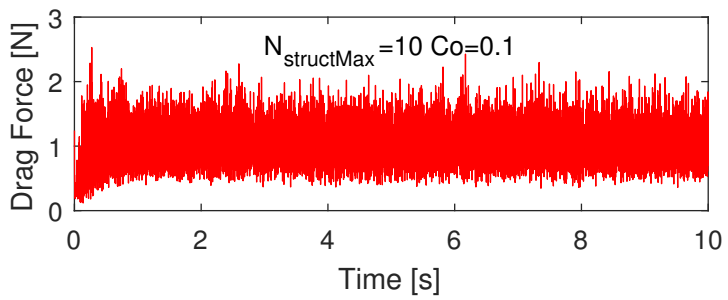

(d)

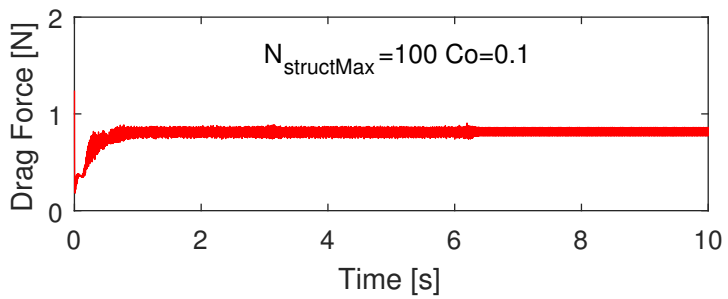

(e)

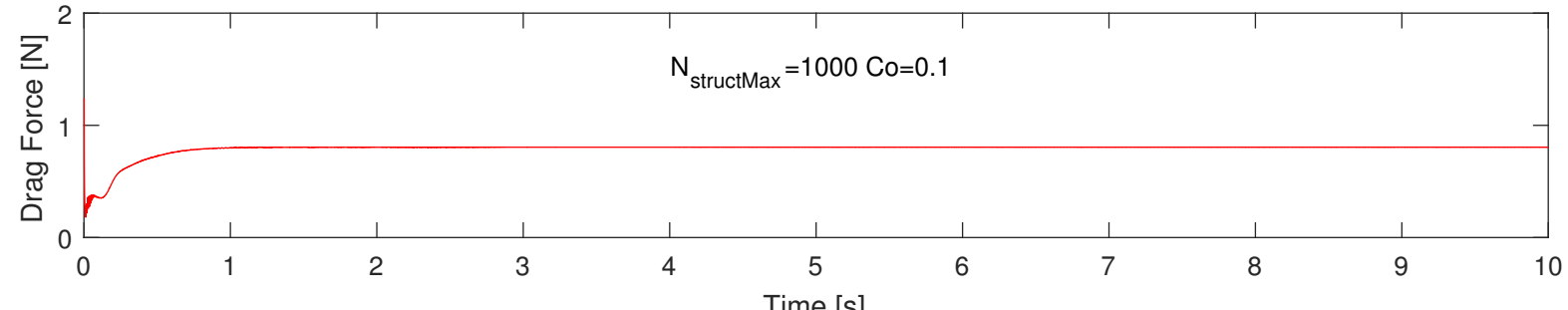

(f)

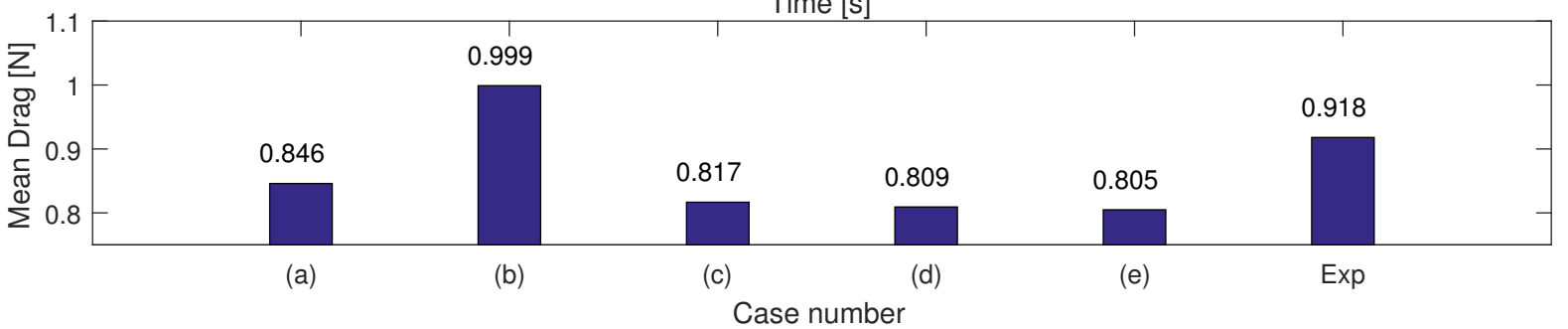

Figure 9: Comparison of time series of the drag force and the mean drag acting on the plane net. Figure (a) - (e) present time series of the drag force for cases with different combinations of Courant number and the number of sub-cycling $N_{\text {struct Max }}$. Figure (f) presents the mean drag force of (a) - (e) and the drag force measured from experiments in Bi et al. (2014b). The mean drag was determined from the time series of the drag force between $4 \mathrm{~s}-10 \mathrm{~s}$. Realizable $k-\epsilon$ turbulence model was applied for all the cases.

the time step for the structural solver. In the numerical model, the quasi-steady assumption was applied. Within one time step, the deformation of the net was evolved under constant hydrodynamic load. But this assumption is not applicable under large time steps, within which the hydrodynamic load is not constant anymore. Otherwise the solution can not be converged to a steady-state solution. Instead, the time series of the force signal and the deformation of the net were oscillating around the mean value, as shown in Fig. 9.

Eliminating/controlling this oscillation is important in some certain cases. For instance, if the net panel is connected with a floater, the motion of the floater should be determined by the instantaneous forces, including the force from the net. The oscillation of the force may lead to instability of the motion of the floater. In unsteady cases such as waves, large amplitude oscillation of the drag force may also cause unreasonable net deformation. Otherwise under steady flow condition, mild amplitude of oscillation as shown in Fig. 9 (c) - (d) is acceptable, since the mean drag force does not deviate much from the experimental result as shown in Fig. 9(f).

Numerical experiments were also carried out to examine the effects of different turbulence models on the drag force of the net panel, and the results were presented in Fig. 10. No significant differences were 

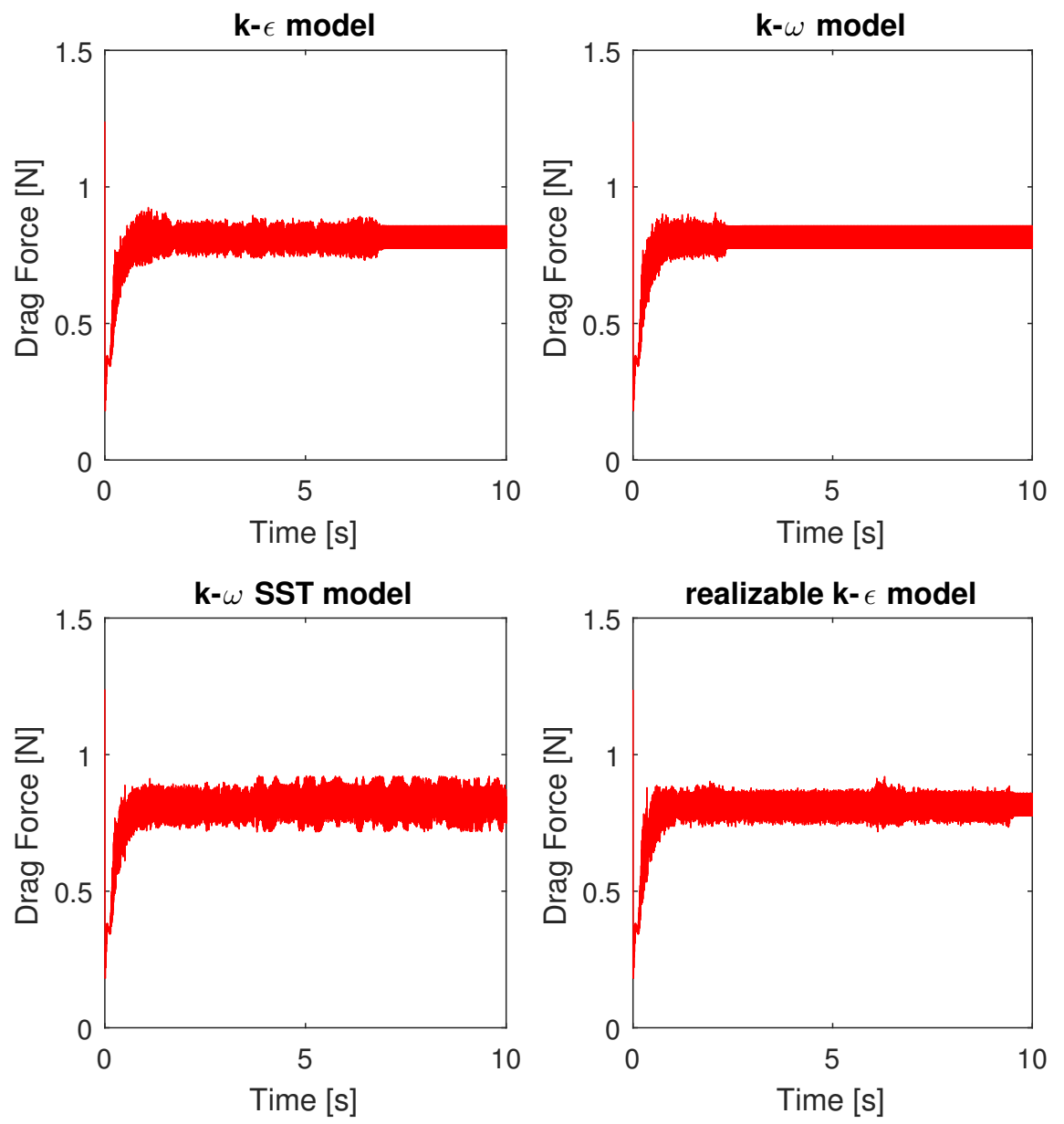

Figure 10: Time series of the drag force on the deformed net panel using $k-\epsilon, k-\omega, k-\omega$ SST and realizable $k-\epsilon$ turbulence models. The Courant number $C o=0.1$, and the sub-cycling number $N_{\text {struct } \operatorname{Max}}=100$.

observed comparing with laminar model. The time series from $k-\omega$ model seems to be quite irregular, and the oscillating amplitude was amplified slightly in the end, while the rest three were finally stabilized and oscillate with constant amplitude.

\subsection{Submerged plane net in waves}

In order to demonstrate the applicability of the numerical model in unsteady conditions, we further validated the numerical model against experiments conducted in Zhao et al. (2008) for submerged plane net in waves. This set of experiments were also carried out in Dalian University of Technology, but the dimension of the wave flume was changed to $69 \times 2 \times 1.8 \mathrm{~m}$. The water depth was set to $1.0 \mathrm{~m}$, while the net was positioned $0.1 \mathrm{~m}$ beneath the still free surface. The plane net was made of Polyethylene and was knotted with a square mesh size of $60 \mathrm{~mm}$ and twine thickness of $1.8 \mathrm{~mm}$. This gave a solidity ratio of 0.059. The net had a length of $0.78 \mathrm{~m}$ and height of $0.6 \mathrm{~m}$. At the bottom, a horizontal bar was attached as the sinker system with a weight of $82 \mathrm{~g}$ in air and diameter of $4 \mathrm{~mm}$.

We set up a 2D wave tank to reproduce the experiment. The general configuration of the domain is shown in Fig. 11. The toolbox developed by Jacobsen et al. (2012) was applied to generate nonlinear stream function waves. The parameters of the selected wave conditions for validation of the numerical model are given in Table 1, where for all the cases the waves are nearly or within the range of linear wave theory. At two ends of the numerical wave tank, two relaxation zones were arranged to generate and absorb the waves. 
Table 1: The parameters of the selected wave conditions for validation

\begin{tabular}{cccc}
\hline Wave case No. & 1 & 2 & 3 \\
\hline Wave period, $T[\mathrm{~s}]$ & 1.40 & 1.40 & 1.40 \\
Wave height, $H[\mathrm{~m}]$ & 0.10 & 0.12 & 0.15 \\
Wave steepness $[-]$ & 0.033 & 0.040 & 0.049 \\
\hline
\end{tabular}

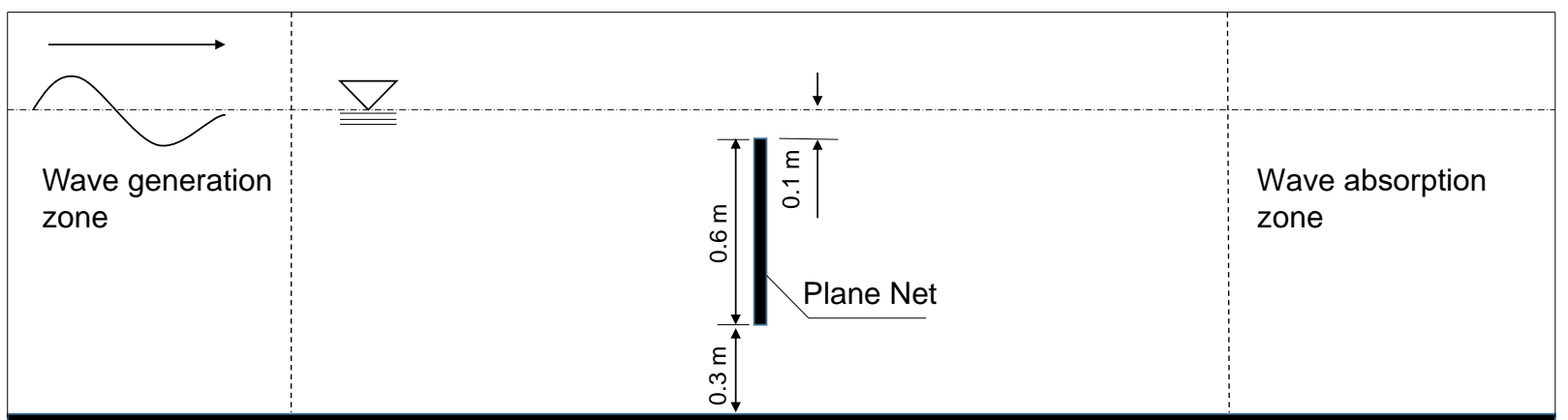

Figure 11: Sketch of the 2D computational domain for simulation of the submerged plane net in waves (unit: $m$ ). The wave was generated in the wave generation zone and propagated from left to right. In the absorption zone the propagating wave was absorbed to avoid reflection. The length of the generation and absorption zone are approximately the same with the wave length. The porous zone representing the plane net structure has a thickness of $20 \mathrm{~mm}$.

The length of each relaxation zone was approximately the same as the wave length. The net was placed in the same position as in the experiment, i.e. $0.1 \mathrm{~m}$ beneath the free surface, and the thickness of the porous zone that represents the net was set to $20 \mathrm{~mm}$.

A snapshot of the free surface wave and the response of the net is given in Fig. 12 for wave case 1. Furthermore, the time series of the drag force on the net panel, the surface elevation at the net position, the displacement of the net panel at bottom and middle point are depicted in Fig. 13. During the simulation, the time step was restricted with $C o=0.1$ and $N_{\text {structMax }}=6000$. This ensures convergence of the result in time domain and a relatively smooth force series.

From Fig. 13 we observe that the surface elevation is leading before the force series, while the displacement of the net panel at the middle and bottom point is lagging behind them. In principle, the crest of surface elevation creates the maximum velocity. If the drag force is the dominant force on the net panel, it should be in phase with the surface elevation. However, this is the case for forces on fixed net structures. For a flexible structure, the deformation of the panel is also an important factor that affects the total force. The inertia force should have minor effect on the net, especially for such kind of low solidity ratio net as illustrated in Chen and Christensen (2016).

The amplitude of the displacement is shown in Fig. 14, where the experimental data from Zhao et al. (2008) is also given for comparison. In general, we observe a better agreement for displacement at the middle point of the net panel, and the displacement at the bottom point was overestimated. One possible error source from the numerical simulation is the $3 \mathrm{D}$ flow effect, since in the experiments the net panel is narrower than the width of the flume. Therefore, 3D flow effect including near wall effect may play a role.

\subsection{Circular cages in current flow}

The third validation case was carried out for 3D flow through and around circular net cages. The experiments have been described in $\mathrm{Bi}$ et al. (2014a), which were conducted in the same flume as the experiment described in Section 4.1. A net made from polyamides was used in the experiments, with the mesh distance $\lambda=20 \mathrm{~mm}$ and $d_{w}=1.2 \mathrm{~mm}$. This gave a solidity ratio of 0.12 . The net cage has a diameter of $0.254 \mathrm{~m}$ and depth of $0.15 \mathrm{~m}$. In the circumferential direction of the cage there were 40 meshes, while in vertical direction there were 8 meshes. The incoming velocities were selected as $0.122,0.178$ and $0.242 \mathrm{~m} / \mathrm{s}$, and the weight of the sinker for all the cases was $8 \mathrm{~g}$ in air. 


$$
t=4 s
$$

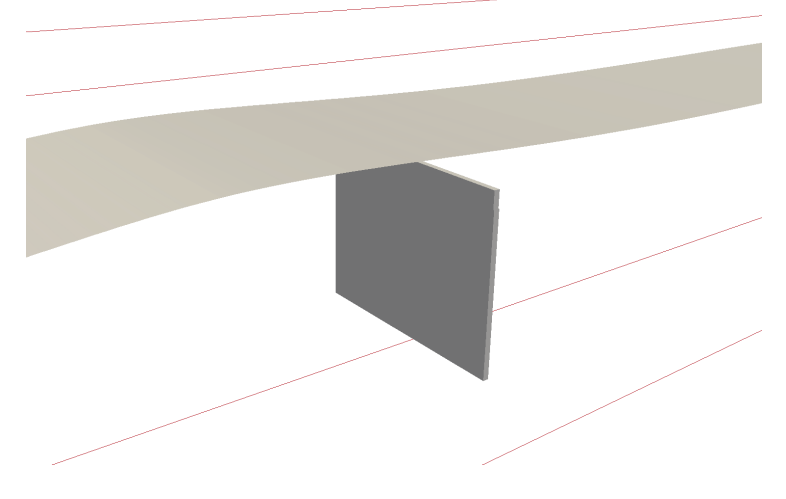

$\mathrm{t}=8 \mathrm{~s}$

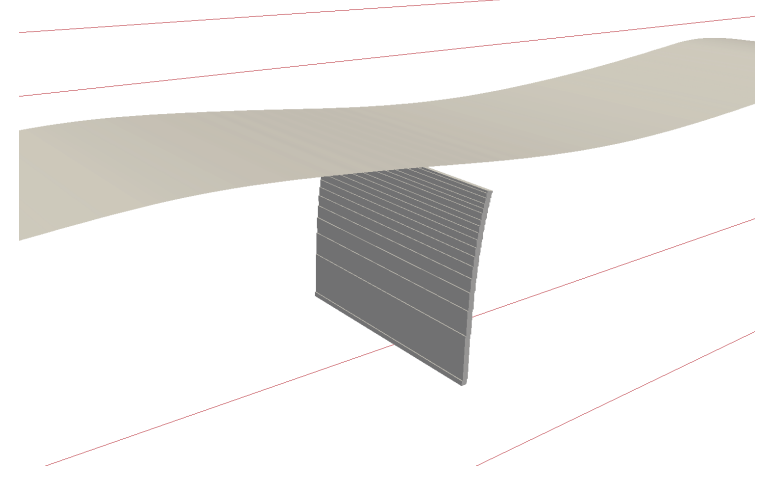

$t=6 \mathrm{~s}$

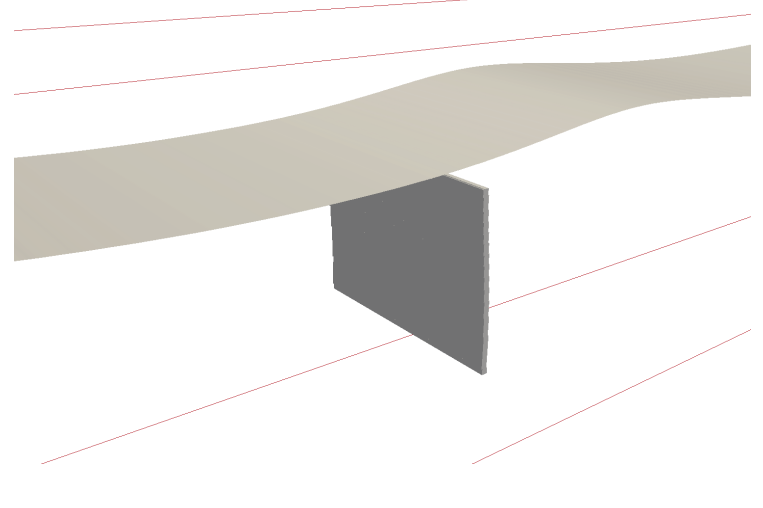

$t=10 s$

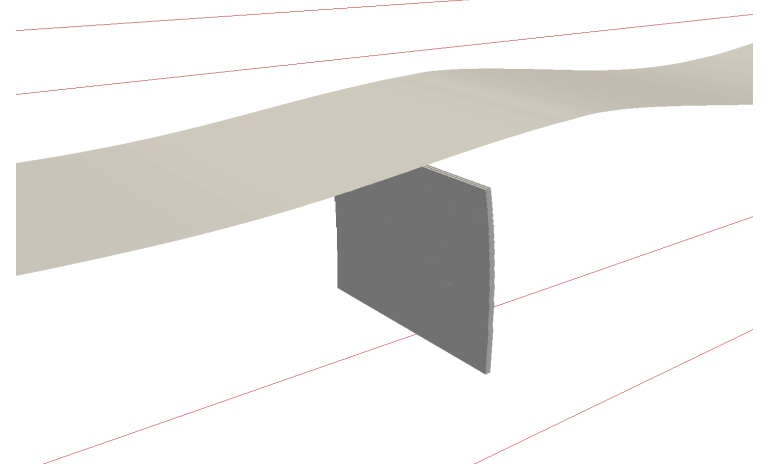

Figure 12: Snapshot of the free surface wave and the deformation of the plane net at different time for wave case 1.

The 3D numerical model was set up corresponding to the experimental setup. The computational domain and the boundary condition are presented in Fig. 15. where the net cage was divided into 16 panels in the circumferential direction and 5 panels in the vertical direction. The solver generated the initial configuration of the net cage based on the input parameters of the net cage, which is shown in Fig. 16. It should be mentioned that in reality, the net cage in still water is not completely vertical due to the sag in the net cage, see Kristiansen and Faltinsen (2015). The diameter at the lower end is approximately 10\% smaller than at the top. But this effect was neglected in the present study, and the initial shape of the net cage was set completely vertical. Furthermore, Fig. 16 also presents the computational mesh, where the mesh in the vicinity of the net cage was refined. This ensures that the porous zones could be updated accurately. The thickness of the porous zones was chosen to be $20 \mathrm{~mm}$, and the corresponding porous resistance coefficients are $C_{1}=9.16 \mathrm{~m}^{-1}, C_{2}=C_{3}=5.67 \mathrm{~m}^{-1}$.

\subsubsection{Cage deformation}

The deformation of the cage is shown in Fig. 17 with the experimental results from Bi et al. (2014a). In addition, the bird's eye view of the cage deformation is given in Fig. 18. In general, the agreement is fair. However, we observe that the curvature at the front part of the cage was not modeled in a correct way, especially close to the bottom. This is due to the effect of the bottom sinker. In the experiment, it was a ring with circular cross section. But in the numerical model, the ring was not resolved by the computational mesh. Instead the forces acting on it were distributed into each lumped mass point. Therefore, this modeling error induced the discrepancy of the curvature between the numerical model and the experiments. Refining the mesh cannot resolve this issue. 

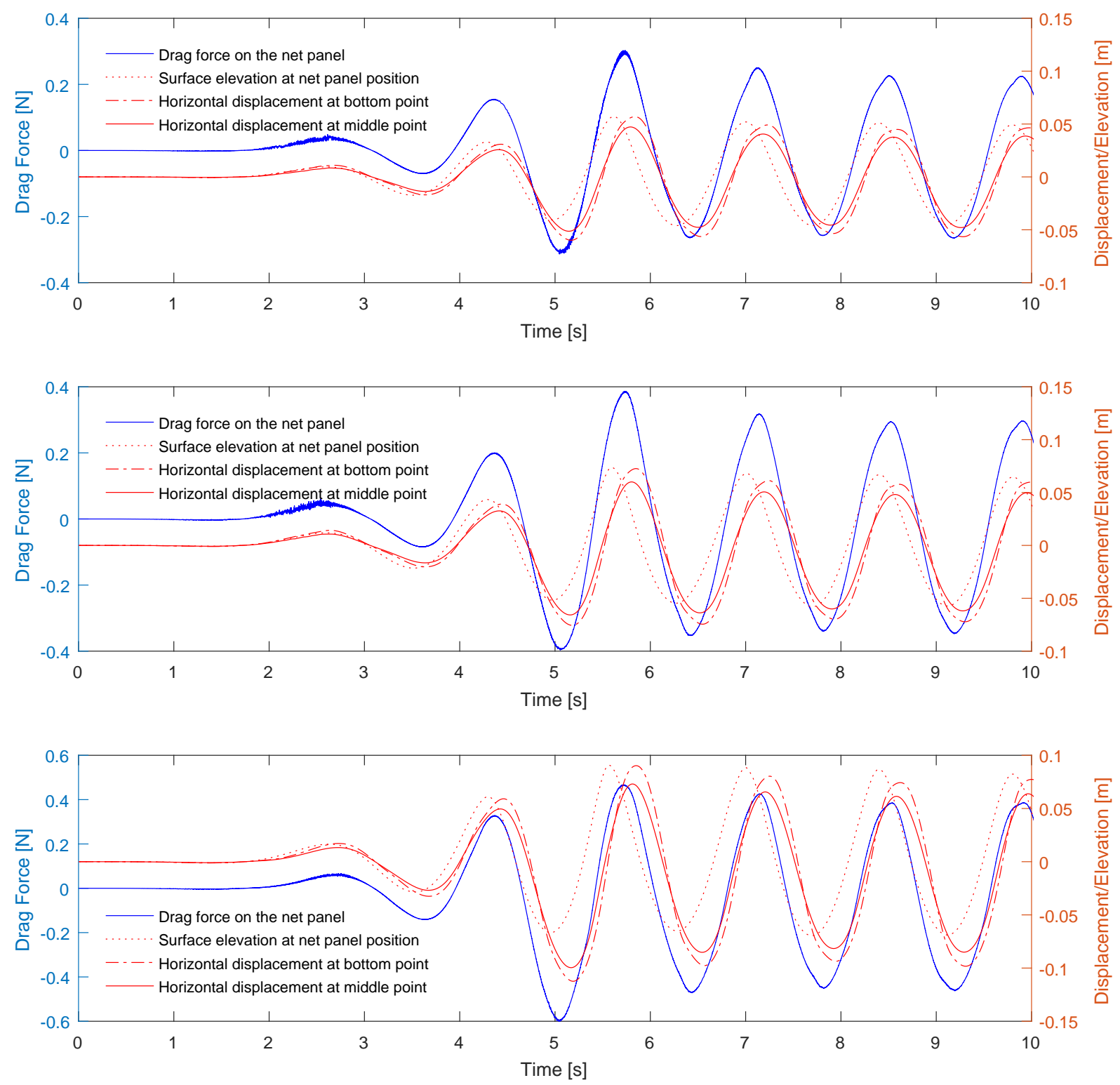

Figure 13: Time series of the drag force on the net panel, surface elevation at the net panel position, horizontal displacement of the net panel at the bottom and middle point. Top: wave case 1. Middle: wave case 2. Bottom: wave case 3. 

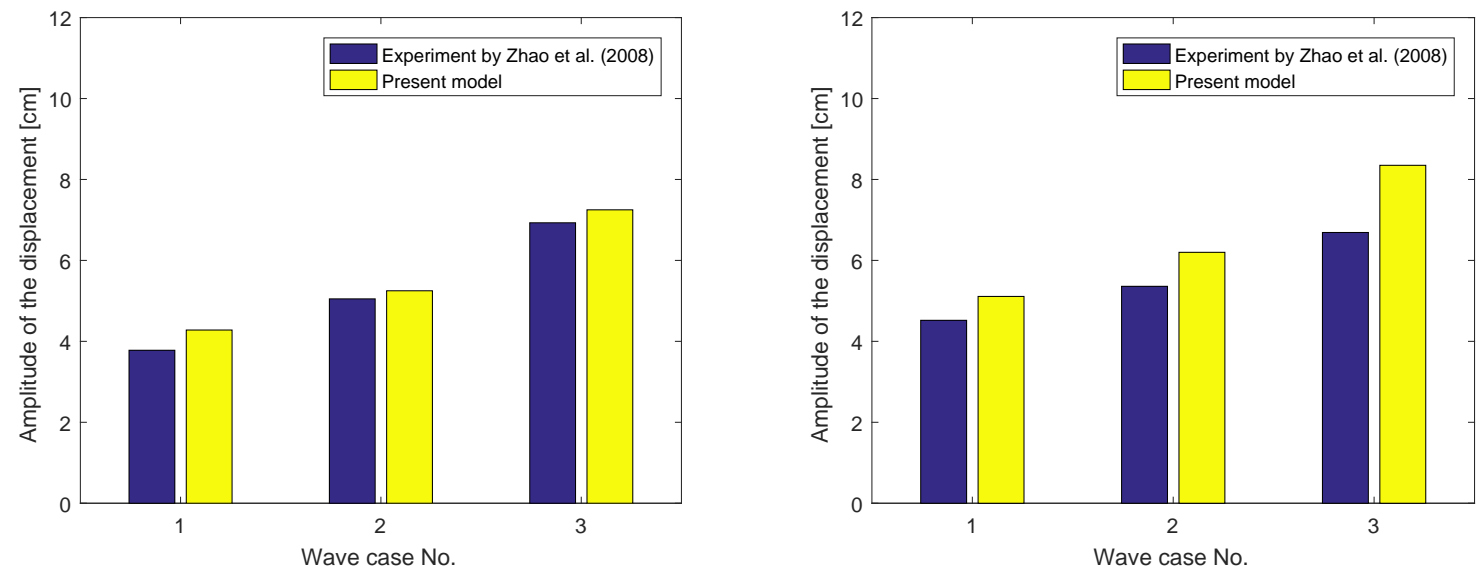

Figure 14: Comparison of the amplitude of the displacement of the net panel between the present numerical model and the experimental data from Zhao et al. (2008). Left: displacement at the middle of the net panel. Right: displacement at the bottom of the net panel.
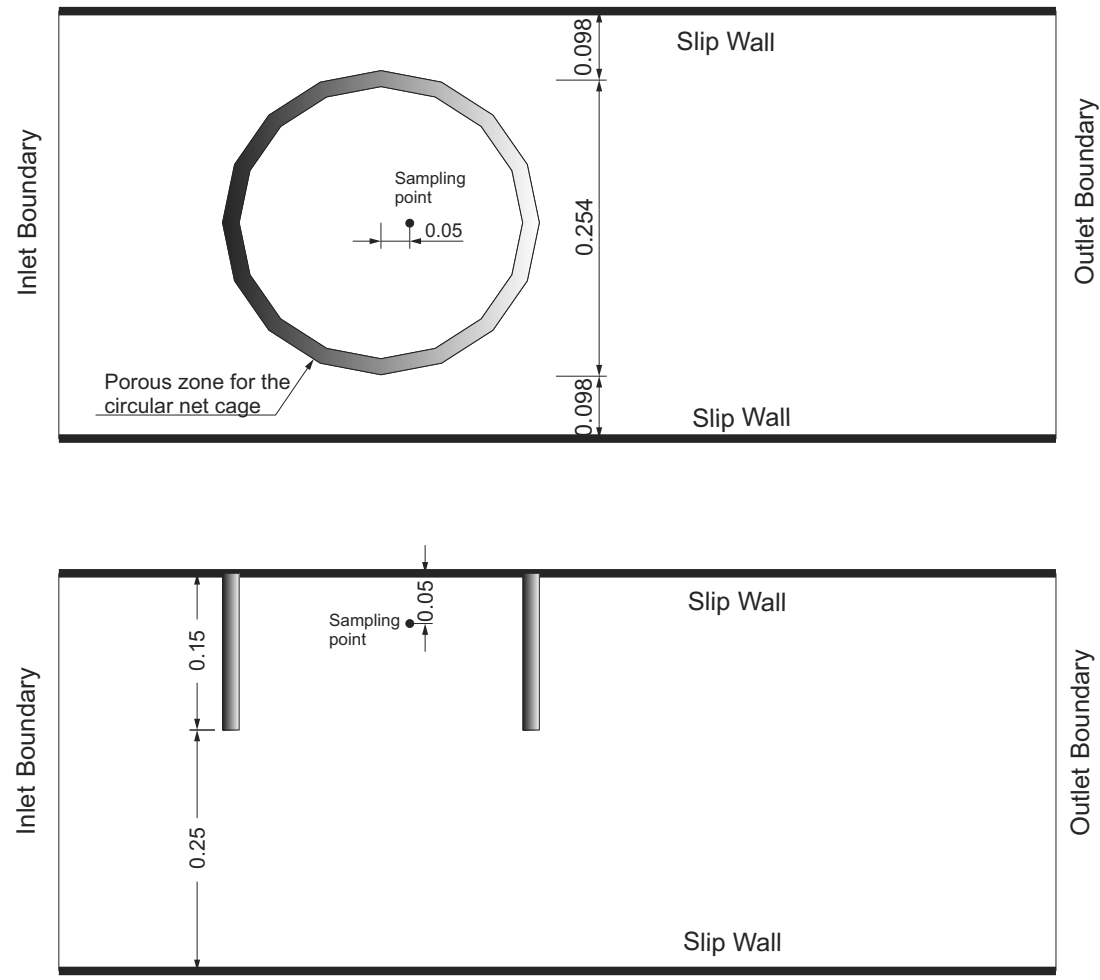

Figure 15: The computational domain and the boundary condition for the simulation of circular net cage in steady current (unit: $\mathrm{m}$ ). Upper: top view. Lower: side view. The boundaries at bottom, top, front and back were modeled as slip walls, and the free surface was not modeled. The porous zone representing the circular net cage has a thickness of $20 \mathrm{~mm}$ and the sinker of the net cage was not modeled. The sampling point is located at $0.05 \mathrm{~m}$ below the free surface and $0.05 \mathrm{~m}$ behind the center of the circular cage. The velocity was sampled at this position and the result is given in Fig. 20 


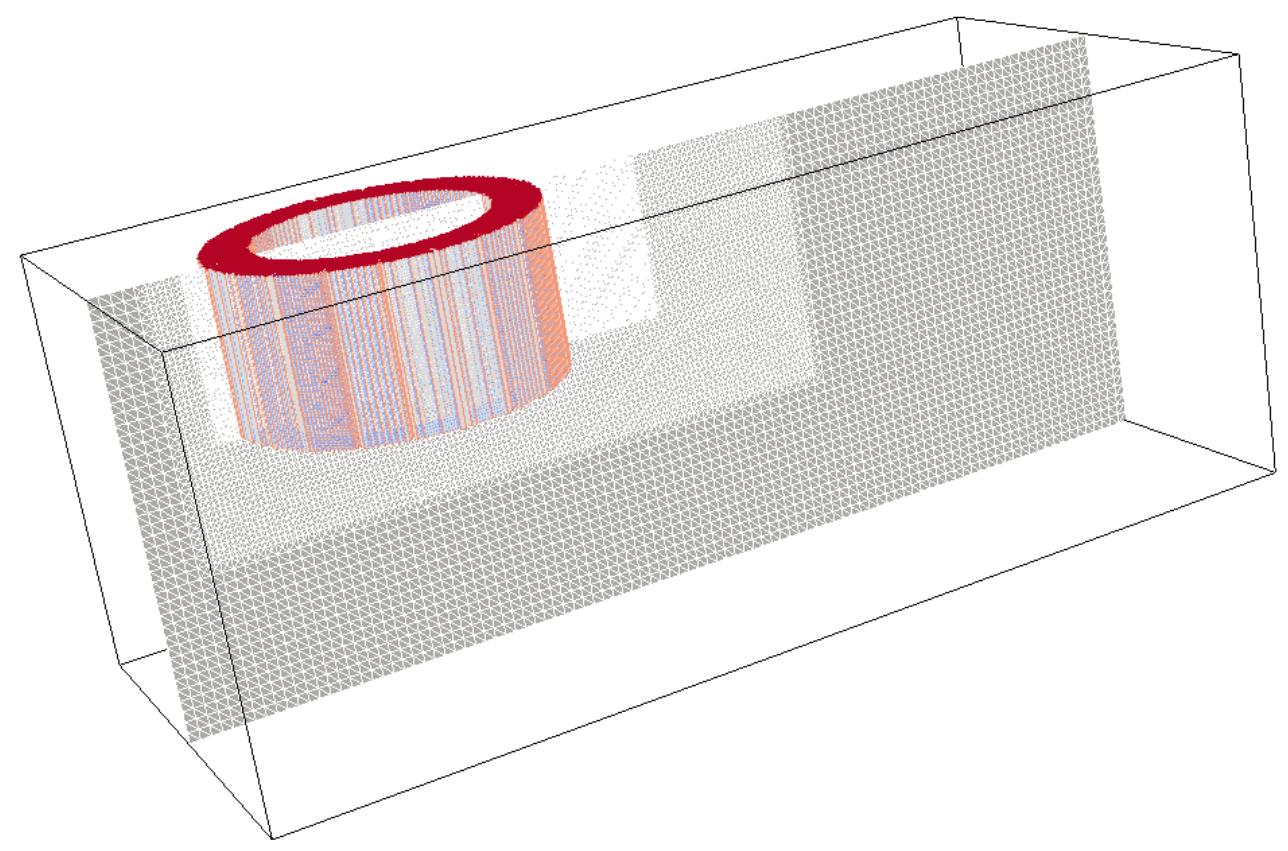

Figure 16: The initial shape of the circular net cage and the computational mesh in the numerical model. The mesh was refined in the vicinity of the circular cage.

\subsubsection{Drag force and downstream velocity}

Comparison of the drag force on the net cage is given in Fig. 19 for different incoming velocities. Similar to the $2 \mathrm{D}$ case as shown in Section 4.1.2, we found a consistent $10 \%-15 \%$ underestimation of the drag force, the reason is unknown yet. In addition, the downstream velocity at the sampling point (the location is given in Fig. 15) is also presented in Fig. 20. It was found that the numerical model can in general produce a better prediction on the downstream velocity. The maximum error occurs for case with $U=0.242 \mathrm{~m} / \mathrm{s}$, where the downstream velocity was overestimated by $9.5 \%$.

\section{Summary and conclusion}

In the present work, we developed a numerical model for analysis of flow through and around aquaculture net cages. The motivation to develop such a model is to consider the deformation of the net cage in the porous media model. The foundation of the work is the model previously developed in Bi et al. (2014a) and Bi et al. (2014b), where the porous media model was coupled with a lumped mass structural model based on the concept of "iteration". We further improved this model by implementing an interface between these two solvers. Therefore, the interaction effects between the net cage and the flow were considered, and the time stepping procedure was introduced. This enables the solver to be applied in both steady and unsteady conditions.

During the development of this model, several characteristics were noticed. The fluid solver allows a relatively large time step (especially for single-phase flow), while the structural solver restricts it to be very small. However, the main computational burden is on the fluid model. The structural model takes much less time per time step. Therefore, in order to reduce the computational time, the global time step was set to the time step for the fluid solver. Quasi steady-state condition was assumed within each time step, and sub-cycling was applied for the structural solver. However, it was observed that both the global time step and the time step for the structural model affect the oscillation of the time series of the load on the net structures. By properly reducing them, the amplitude of the oscillation can be reduced or even eliminated. 

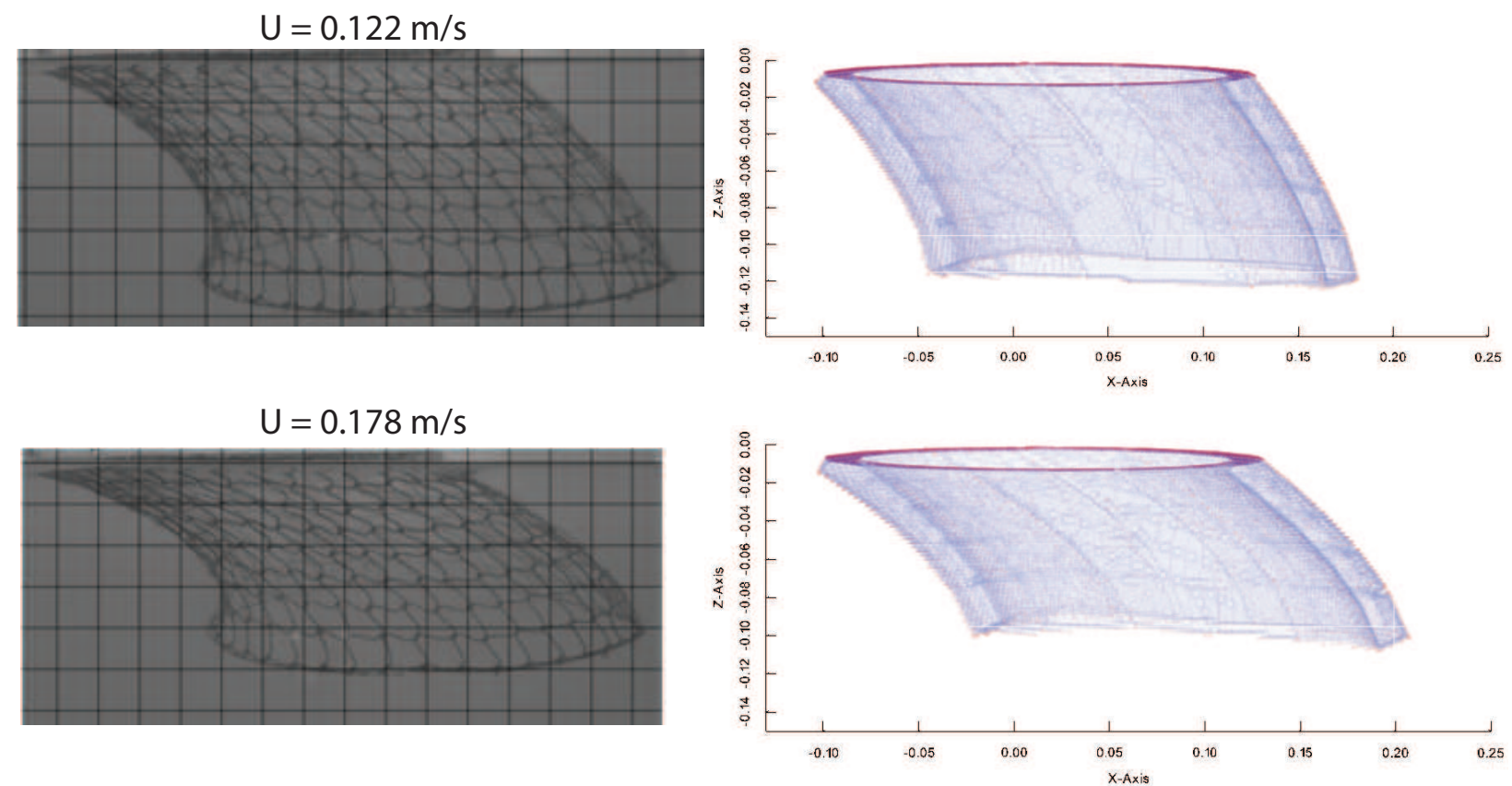

\section{$\mathrm{U}=0.242 \mathrm{~m} / \mathrm{s}$}
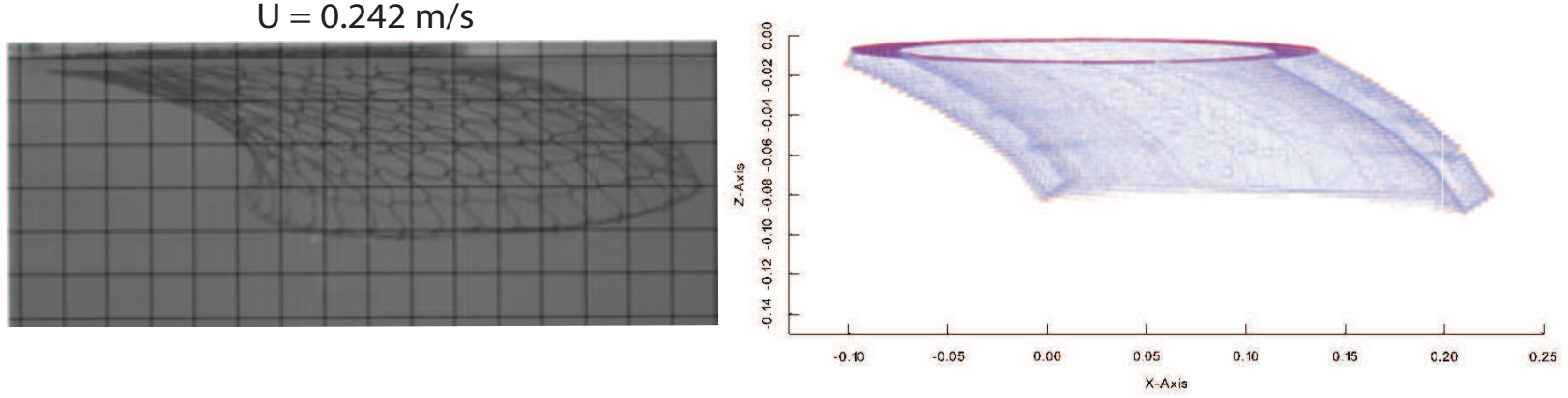

Figure 17: Comparison of the steady-state shape of the circular net cage between the present numerical calculations (right) and the experimental results (left) in Bi et al. (2014a) for incoming velocities of $0.122,0.178$ and $0.242 \mathrm{~m} / \mathrm{s}$. The weight of the sinker is $8 \mathrm{~g}$ in air, and the solidity ratio $S_{n}=0.12$. The figures in the left were reproduced from Fig. 10 in Bi et al. (2014a), where the side length of the mesh is $25 \mathrm{~mm}$.

$$
\mathrm{U}=0.122 \mathrm{~m} / \mathrm{s}
$$

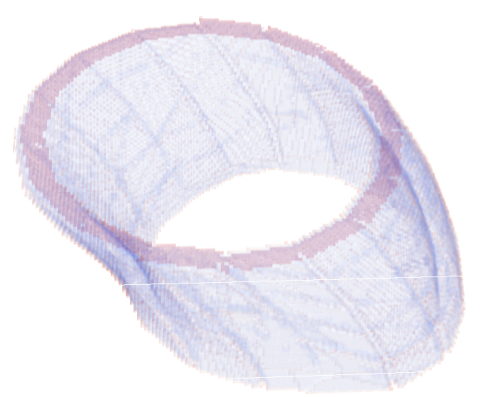

$$
\mathrm{U}=0.178 \mathrm{~m} / \mathrm{s}
$$

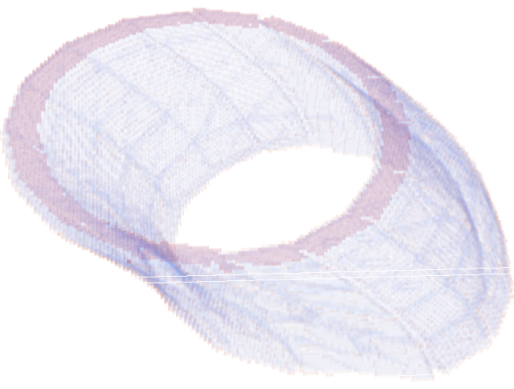

$\mathrm{U}=0.242 \mathrm{~m} / \mathrm{s}$

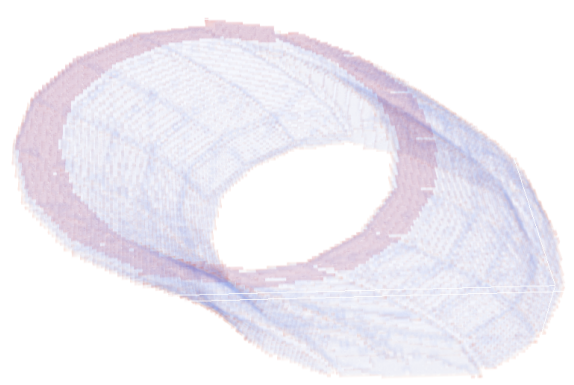

Figure 18: Bird's eye view of the same simulation as presented in Fig. 17. The current direction is from left to right. 


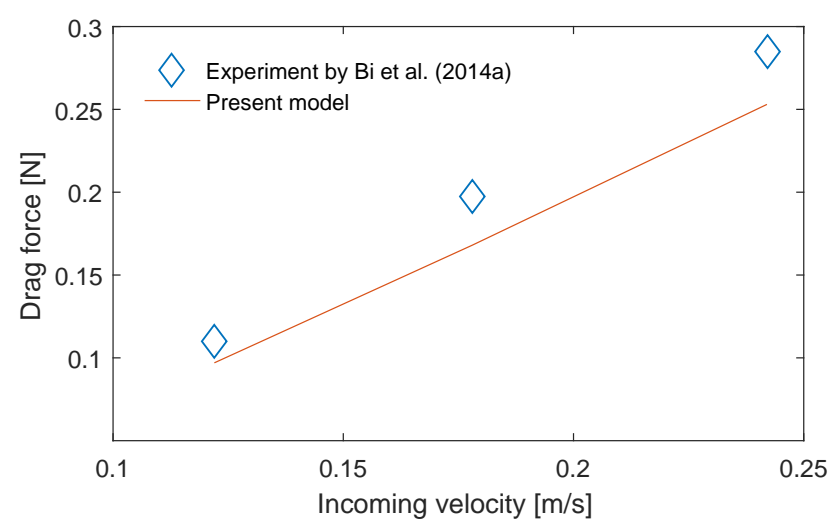

Figure 19: Results from the present numerical model vs. experiments by Bi et al. (2014a) on the drag force of the circular deformed net cages.

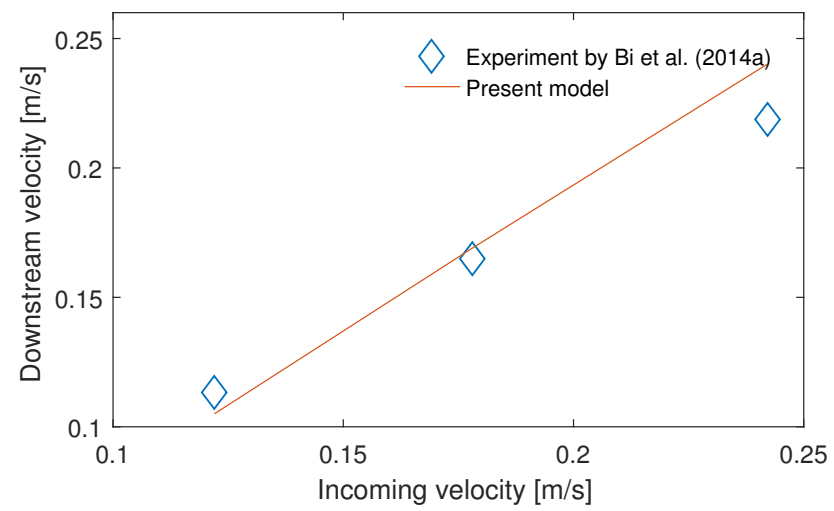

Figure 20: Results from the present numerical model vs. experiments by Bi et al. (2014a) on the downstream velocity at the sampling point as shown in Fig. 15

The numerical model was validated against three sets of experiments, for plane net in steady current and regular waves, and for circular cages in steady current. In all the experiments, the net was top fixed and bottom weighted. The deformation of the net, the velocity at downstream and the drag forces were compared with the experimental data for plane net and circular cage in steady current. In general, the agreement was fair, but we found a consistent underestimation of the drag force. For regular wave interaction with plane net panel, the amplitude of the displacement was compared with the experimental results at the bottom and middle of the net panel. A better prediction was given for the middle point, while at the bottom point the displacement was overestimated.

Future work will be focused on floater-net interaction effects. The floating rings will be incorporated in the numerical model, and its rigid body motion will be solved by a six degree of freedom motion solver. Therefore, the top layer of the net cages will not be fixed, but follow the motion of the ring.

\section{Acknowledgement}

The authors would like to thank Dr. Chunwei Bi from Dalian University of Technology for kindly providing the experimental data and the relative figures. Furthermore, Prof. Trygve Kristiansen from NTNU is acknowledged for the discussions on turbulent effects and for hosting the first author during the preparation of this paper. The research was partially supported by FP7-OCEAN-2011 project Innovative multi-purpose offshore platforms: planning, design and operation, MERMAID, 288710, under the call Ocean of Tomorrow. 


\section{Appendix A. Update of the porous zones based on the nodal position of the structural element}

This section gives an introduction on how to update the porous zones based on the transferred nodal position from the structural model. As mentioned in Section 3.2, the idea is to first empty the existing porous zones, then identify the cells that belong to the updated porous zones. Below, the criteria is derived to judge if one cell is within the updated porous zones.

\section{$2 D$ case}

Fig. 21 gives an example on the mapping between the fluid and structural elements in 2D cases. Herein, the nodes in the structural elements are in the intersection between the panel elements. Hereby one structural element is composed of two nodes and one plane panel element. The corresponding porous zone in the fluid model is generated based on the structural elements and the thickness of the porous zone $t_{0}$. For an arbitrary mesh cell, given that the cell center is $\boldsymbol{x}$, and the position of Node 1 and Node 2 are $\boldsymbol{x}_{1}$ and $\boldsymbol{x}_{2}$. Then the corresponding length $d_{1}, d_{2}$ and $l$ in Fig. 21 can be calculated as:

$$
\begin{gathered}
d_{1}=\operatorname{mag}\left(\boldsymbol{x}-\boldsymbol{x}_{\mathbf{1}}\right) \\
d_{2}=\operatorname{mag}\left(\boldsymbol{x}-\boldsymbol{x}_{\mathbf{2}}\right) \\
l=\operatorname{mag}\left(\boldsymbol{x}_{\mathbf{1}}-\boldsymbol{x}_{\mathbf{2}}\right)
\end{gathered}
$$

where mag defines the magnitude of the vector. Recalling the Pythagorean theorem, the following relation was deduced:

$$
l=\sqrt{d_{1}^{2}-d^{2}}+\sqrt{d_{2}^{2}-d^{2}}
$$

By some mathematical manipulation, the distance $d$ was expressed as:

$$
d=\sqrt{d_{1}^{2}-\frac{1}{4 l^{2}}\left(d_{1}^{2}-d_{2}^{2}+l^{2}\right)^{2}}
$$

The angle $\cos \alpha_{1}$ and $\cos \alpha_{2}$ denoted in Fig. 21 were calculated as:

$$
\begin{aligned}
& \cos \alpha_{1}=\left(\boldsymbol{x}-\boldsymbol{x}_{\mathbf{1}}\right) \cdot\left(\boldsymbol{x}_{\mathbf{2}}-\boldsymbol{x}_{\mathbf{1}}\right) /\left(\operatorname{mag}\left(\boldsymbol{x}-\boldsymbol{x}_{\mathbf{1}}\right) \operatorname{mag}\left(\boldsymbol{x}_{\mathbf{2}}-\boldsymbol{x}_{\mathbf{1}}\right)\right) \\
& \cos \alpha_{2}=\left(\boldsymbol{x}-\boldsymbol{x}_{\mathbf{2}}\right) \cdot\left(\boldsymbol{x}_{\mathbf{1}}-\boldsymbol{x}_{\mathbf{2}}\right) /\left(\operatorname{mag}\left(\boldsymbol{x}-\boldsymbol{x}_{\mathbf{2}}\right) \operatorname{mag}\left(\boldsymbol{x}_{\mathbf{1}}-\boldsymbol{x}_{\mathbf{2}}\right)\right)
\end{aligned}
$$

By looping through all the cells, the quantities listed above for each cell were examined. Two criteria needs to be satisfied if one cell belongs to the porous zone: (1) $d<1 / 2 t_{0}$. (2) $\alpha_{1}<\pi / 2, \alpha_{2}<\pi / 2$.

\section{$3 D$ case}

In 3D case, in the lumped mass structural model, each element is associated with four nodes, and they are located at the corners of the structural element as depicted in Fig. 2. However, the four nodes are not necessarily in the same plane. Therefore, to ease the mapping between the elements, the rectangular element was split into two triangular elements. Each element is composed of three nodes and one panel element. In the fluid model, the corresponding porous zone was generated by extending the plane where the structural element is within into a prism with thickness $t_{0}$.

Fig. 22 depicts how the elements in structural and fluid model are mapped in 3D case. Here $d$ is the distance between the panel element and the cell center. It was calculated based on the normal vector of the plane $\boldsymbol{n}$, which was based on the cross product of the arbitrary two in-plane vectors, e.g. $\boldsymbol{x}_{1}-\boldsymbol{x}_{\mathbf{2}}$ and $\boldsymbol{x}_{2}-\boldsymbol{x}_{3}$, where $\boldsymbol{x}_{1}, \boldsymbol{x}_{2}$ and $\boldsymbol{x}_{3}$ are the position vector of Node $1-3$. But this normal vector has to be in the same half space with the point $\boldsymbol{x}$. Then $d$ was calculated as

$$
d=\left(\boldsymbol{x}-\boldsymbol{x}_{\mathbf{2}}\right) \cdot \boldsymbol{n}
$$




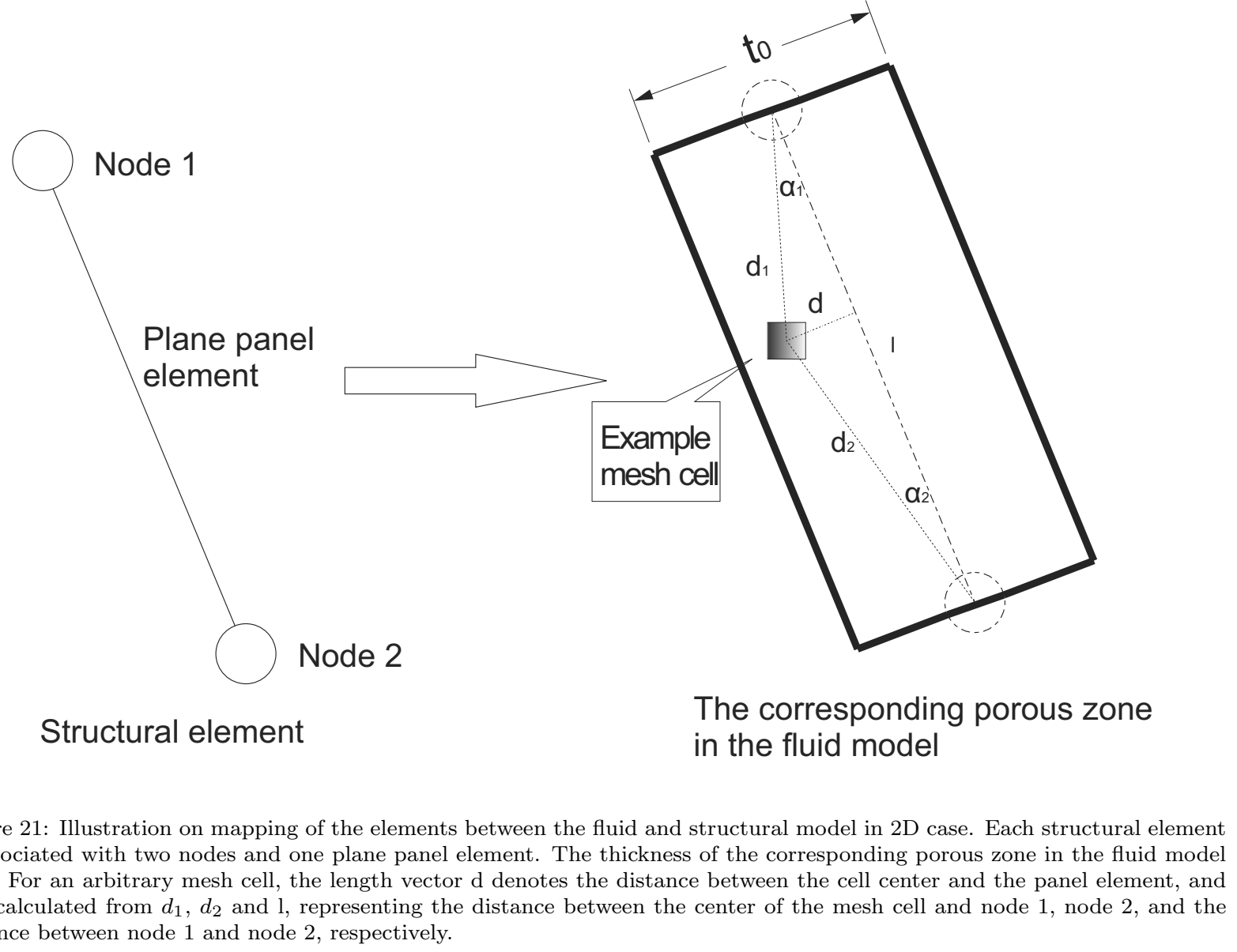

Therefore the position for the perpendicular foot $\boldsymbol{x}_{\mathbf{0}}$ was obtained as:

$$
\boldsymbol{x}_{\mathbf{0}}=\boldsymbol{x}-d \boldsymbol{n}
$$

If one cell is within the porous zone, two conditions need to be satisfied: (1) $d<1 / 2 t_{0}$. (2) $x_{0}$ is within the triangular $x_{1} x_{2} x_{3}$. If $\boldsymbol{x}_{\mathbf{0}}$ is within the triangular $x_{1} x_{2} x_{3}$, the total area of the triangular $x_{0} x_{1} x_{2}, x_{0} x_{1} x_{3}$ and $x_{0} x_{2} x_{3}$ should be equal to the area of the triangular $x_{1} x_{2} x_{3}$, where an error of machine precision was allowed. If both $d$ and $\boldsymbol{x}_{0}$ satisfied the conditions, the cell belongs to the porous zone.

\section{Appendix B. Analytical solution of the mixing layer flow based on boundary layer theory}

Mixing layer flow is a kind of free shear flow, where there are no walls directly at the flow. The general configuration for mixing layer flow is given in Fig. 23. Initially there exist two parallel and unperturbed free streams with different velocity $U$ and $\lambda U(0<\lambda<1)$. With the development of the flow, two streams interact with each other. Due to friction forces, a thin mixing zone is produced where the transition takes from $U$ to $\lambda U$.

We emphasize that the transition zone is the region of space where a solution is sought. Particularly in the present work, the longitudinal velocity distribution in the transition zone is of interest. The transition zone itself is slender, where the transverse velocity component is small compared with the longitudinal velocity component. Therefore, the Prandtl boundary layer equation is valid for the flow in this zone, and is used to seek the solution. A complete and comprehensive derivation has been given in e.g. Schlichting and Gersten (2000) or Lessen (1949). Below, we briefly summarize the derivation process. 


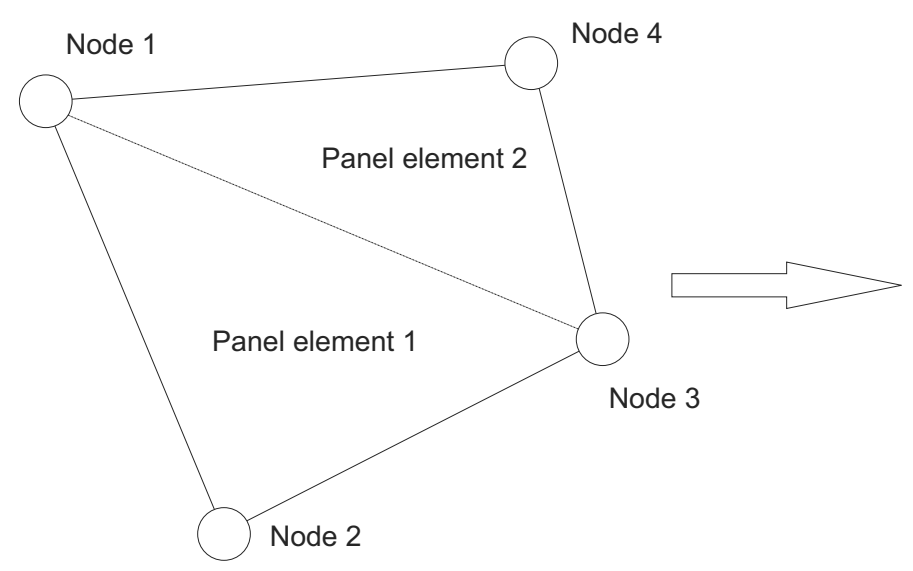

Structural element 1 and 2

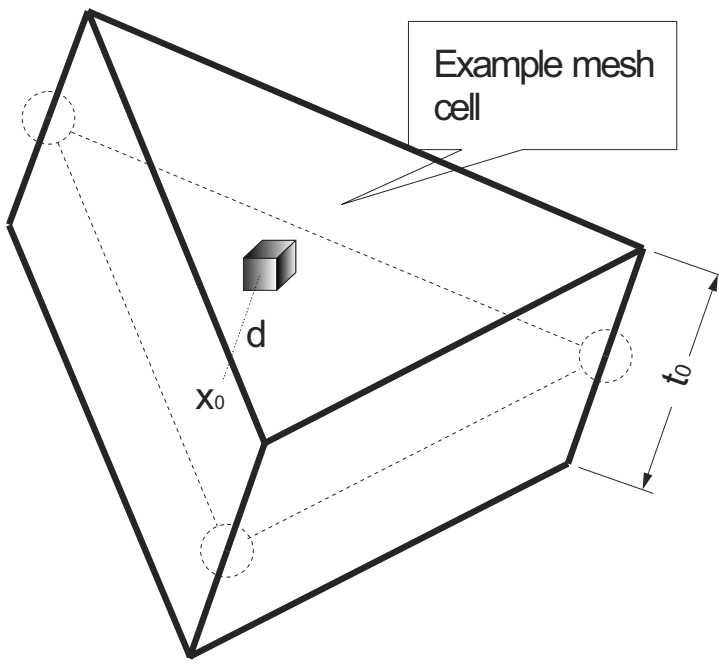

The porous zone in the fluid model corresponding to structural element 1

Figure 22: Illustration on mapping of the elements between the fluid and structural model in 3D case. Each rectangular structural element was split into two triangular element, which is composed of one panel element and three nodes. The thickness of the corresponding porous zone in the fluid model is $t_{0}$. For an arbitrary mesh cell, the length vector d denotes the distance between the panel element and the cell center. $x_{0}$ is the perpendicular foot within the panel plane.

\section{Laminar mixing layer flow}

The starting point was the Prandtl boundary layer equation for the laminar plane flow:

$$
\begin{gathered}
\frac{\partial u}{\partial t}+u \frac{\partial u}{\partial x}+v \frac{\partial u}{\partial \bar{y}}=-\frac{1}{\rho} \frac{\partial P}{\partial x}+\nu \frac{\partial^{2} u}{\partial y^{2}} \\
\frac{\partial u}{\partial x}+\frac{\partial v}{\partial y}=0
\end{gathered}
$$

where the velocity vector $\boldsymbol{u}$ was decomposed into $u$ and $v$ in Cartesian coordinate system, $\nu$ is the kinematic viscosity. The boundary layer system is related to the outer flow as follows:

$$
\frac{\partial U}{\partial t}+U \frac{\partial U}{\partial x}=-\frac{1}{\rho} \frac{\partial P}{\partial x}
$$

where $U$ is the velocity in the outer flow (the flow outside the boundary layer).

The governing equations for the plane boundary layer given above were further simplified for mixing layer flow. Considering that the system is steady, the derivative with regard to time should be zero. In addition, the velocity of free stream at the positive and negative infinity is constant. Hereby the pressure gradient in longitudinal direction is zero. Therefore, Eq. B.1- B.2. were reduced to:

$$
\begin{gathered}
u \frac{\partial u}{\partial x}+v \frac{\partial u}{\partial y}=\nu \frac{\partial^{2} u}{\partial y^{2}} \\
\frac{\partial u}{\partial x}+\frac{\partial v}{\partial y}=0
\end{gathered}
$$

The basic assumption for application of Eq. B.4-B.5 on mixing layer flow is that the velocity profiles at different points are affine or similar. This is the so-called similar solution where the velocity profiles can 


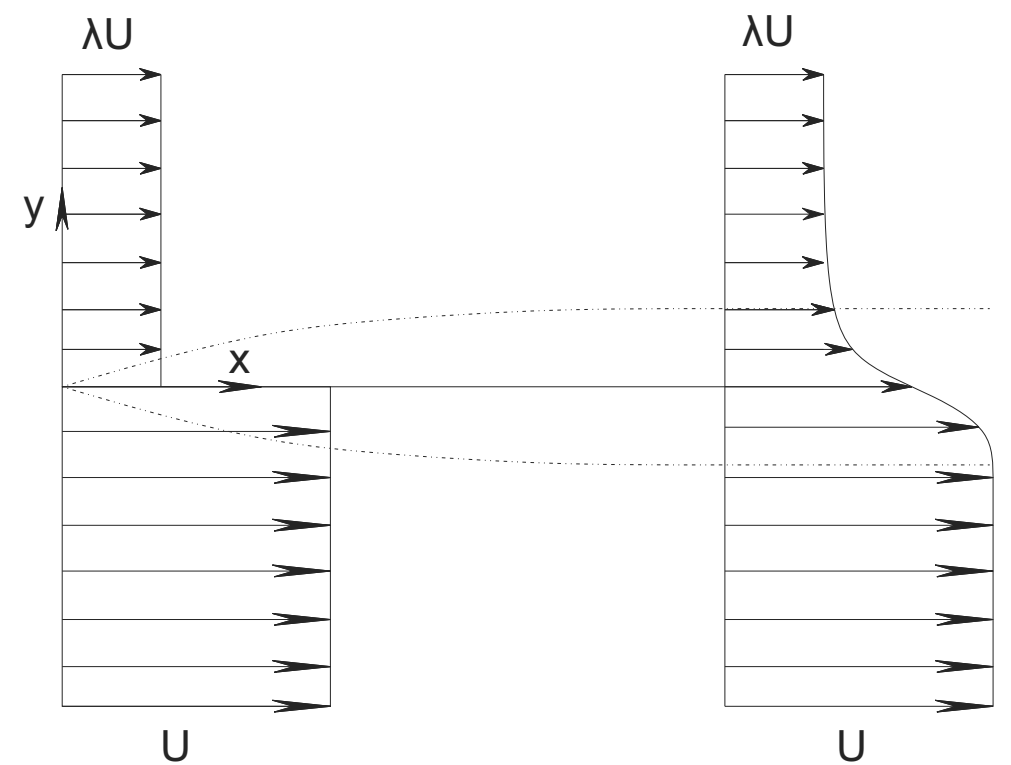

Figure 23: The velocity profiles in the mixing layer. The left profile is at the initial state with two unperturbed parallel flows with velocities $U$ and $\lambda U$. The right profile is after some development where two parallel flows interact with each other. A thin mixing zone is produced in-between the parallel flows as denoted by the dash line.

be mapped onto one another by choosing suitable scaling factors. This means that the normalized velocity $u / U$ is independent of $x$. (The velocity at negative infinite $U$ was selected as the reference velocity for normalization.) Actually it is only a function of $\eta$, which was transformed from $y$ as:

$$
\eta=\frac{y}{\delta(x)}
$$

Here $\delta(x)$ is denoted as a scaled measure of the boundary layer thickness, see Schlichting and Gersten (2000). By dimensional analysis it has the following form:

$$
\delta(x) \sim \sqrt{\frac{x \nu}{U}}
$$

Hereby $\eta$ was reformulated as

$$
\eta=y \sqrt{\frac{U}{2 \nu x}}
$$

Hereafter, we introduce the trial solution for the stream function $\psi$

$$
\psi=\sqrt{2 \nu x U} f(\eta)
$$

where $f(\eta)$ is the nondimensional stream function, and is independent of $x$. Then the velocity components were related to the stream function $\psi$ as

$$
\begin{gathered}
u=\frac{\partial \psi}{\partial y}=\frac{\partial \psi}{\partial \eta} \frac{\partial \eta}{\partial y}=U f^{\prime}(\eta) \\
v=-\frac{\partial \psi}{\partial x}=-\frac{\partial \psi}{\partial \eta} \frac{\partial \eta}{\partial x}=\sqrt{\frac{\nu U}{2 x}}\left(\eta f^{\prime}-f\right)
\end{gathered}
$$


By substituting Eq. B.10-B.11 into Eq. B.4, an ordinary differential equation was obtained

$$
\frac{\mathrm{d}^{3} f}{\mathrm{~d} \eta^{3}}+f \frac{\mathrm{d}^{2} f}{\mathrm{~d} \eta^{2}}=0
$$

with the boundary conditions

$$
\eta \rightarrow+\infty: \frac{\mathrm{d} f}{\mathrm{~d} \eta}=\lambda ; \quad \eta \rightarrow-\infty: \frac{\mathrm{d} f}{\mathrm{~d} \eta}=1
$$

Eq. B.12 is a third order differential equation. Therefore, an additional boundary condition is needed. Ting (1959) has shown that based on the global balance of momentum, the third boundary condition was obtained as

$$
v_{-\infty}=-\lambda v_{\infty}
$$

Recall that the velocity $v$ was given in Eq. B.11). Therefore, Eq. B.14 was rewritten as

$$
\lim _{\eta \rightarrow-\infty}\left(\eta \frac{\mathrm{d} f}{\mathrm{~d} \eta}-f\right)=-\lambda \lim _{\eta \rightarrow \infty}\left(\eta \frac{\mathrm{d} f}{\mathrm{~d} \eta}-f\right)
$$

It is observed that Eq. B.12) does not explicitly contain $\eta$. Hereby in Schlichting and Gersten (2000) it is suggested to apply the following equation as the third boundary condition which is actually derived from Eq. (B.14

$$
f(0)=0
$$

In summary, Eq. (B.12 - B.13) and Eq. $\mathrm{B} .16$ are the equation with the boundary conditions that were solved for the laminar mixing layer flow. In combination with Eq. (B.10), the velocity profile $u$ in the transition zone was retrieved.

\section{Turbulent mixing layer flow}

Regarding turbulent mixing layer flow, one distinct characteristic is that turbulent effects start to play a role. For turbulent free shear flow including the turbulent mixing layer flow, due to absence of the wall, turbulent effects are completely dominant over the molecular viscosity. Therefore, the governing equations were slightly changed as below

$$
\begin{gathered}
\bar{u} \frac{\partial \bar{u}}{\partial x}+v \frac{\partial \bar{u}}{\partial y}=\nu_{t} \frac{\partial^{2} \bar{u}}{\partial y^{2}} \\
\frac{\partial \bar{u}}{\partial x}+\frac{\partial \bar{v}}{\partial y}=0
\end{gathered}
$$

where $\bar{u}$ and $\bar{v}$ are Reynolds averaged velocity components. $\nu_{t}$ is the turbulent viscosity. Note that the molecular viscosity has been neglected as mentioned above.

Readers are referred to e.g. Schlichting and Gersten (2000) for detailed derivation on the governing equations for turbulent mixing layer flow based on Eq. (B.17- B.18). But it will be neglected presently. The final derived equations have the same form as the laminar one, as given in Eq. B.12 - B.13) and Eq. B.16. However, the nondimensional variable $\eta$ has different meaning as

$$
\eta=\frac{y}{\Delta}
$$

where $\Delta$ is the extension of the turbulent free shear layer in the transversal direction, which was expressed as

$$
\Delta=\alpha x
$$

where $\alpha$ was a slenderness parameter, which can be interpreted as the inverse of the characteristic turbulent Reynolds number. It was empirically predicted as a function of $\lambda$

$$
\alpha(\lambda)=\alpha_{0} \frac{1-\lambda}{\sqrt{1+\lambda}}
$$

where $\alpha_{0}=0.045$. 


\section{References}

Berberović, E., van Hinsberg, N., Jakirlić, S., Roisman, I., Tropea, C., 2009. Drop impact onto a liquid layer of finite thickness: Dynamics of the cavity evolution. Physical Review E 79, 036306. URL: http://link.aps.org/doi/10.1103/PhysRevE.79. 036306 doi 10.1103/PhysRevE.79.036306

Bi, C., Zhao, Y., Dong, G., Zheng, Y., Gui, F., 2014a. A numerical analysis on the hydrodynamic characteristics of net cages using coupled fluid-structure interaction model. Aquacultural Engineering 59, 1-12. URL: http://linkinghub.elsevier. com/retrieve/pii/S014486091400003Xhttp://ww.sciencedirect.com/science/article/pii/S014486091400003X doi 10 . $1016 / j$. aquaeng. 2014.01 .002

Bi, C.W., Zhao, Y.P., Dong, G.H., Xu, T.J., Gui, F.K., 2013. Experimental investigation of the reduction in flow velocity downstream from a fishing net. Aquacultural Engineering 57, 71-81. URL: http://linkinghub.elsevier.com/retrieve/ pii/S0144860913000691 doi 10.1016/j.aquaeng.2013.08.002.

Bi, C.W., Zhao, Y.P., Dong, G.H., Xu, T.J., Gui, F.K., 2014b. Numerical simulation of the interaction between flow and flexible nets. Journal of Fluids and Structures 45, 180-201. URL: http://linkinghub.elsevier.com/retrieve/pii/ S0889974613002594 doi $10.1016 / \mathrm{j} \cdot \mathrm{jfluidstructs.2013.11.015}$

Chen, H., Christensen, E.D., 2016. Investigations on the porous resistance coefficients for fishing net structures. Journal of Fluids and Structures 65, 76-107. URL:/http://www.sciencedirect.com/science/article/pii/S0889974615301687, doi 10. $1016 / j \cdot j$ fluidstructs.2016.05.005

Devilliers, M., Vincent, B., Mnassri, I., 2016. A new adaptive mesh refinement to model water flow around fishing nets. Ocean Engineering 113, 34-43. URL: http://www.sciencedirect.com/science/article/pii/S0029801815006630, doi 10.1016/j. oceaneng.2015.12.009

Hou, G., Wang, J., Layton, A., 2012. Numerical Methods for Fluid-Structure Interaction - A Review. Communications in Computational Physics 12, 337-377. URL: http://www.global-sci.com/issue/abstract/readabs.php?vol=12\{\&\}page= $337\{\&\}$ issue $=2\{\&\}$ ppage $=377\{\&\}$ year $=2012$ doi $10.4208 /$ cicp $.291210 .290411 \mathrm{~s}$

Hübner, B., Walhorn, E., Dinkler, D., 2004. A monolithic approach to fluid-structure interaction using space-time finite elements. Comput. Methods Appl. Mech. Engrg. 193, 2087-2104. doi 10.1016/j.cma.2004.01.024

Issa, R.I., 1986. Solution of the implicitly discretised fluid flow equations by operator-splitting. J. Comput. Phys. 62 , 40-65. URL: http://dx.doi.org/10.1016/0021-9991(86)90099-9 doi 10.1016/0021-9991(86)90099-9

Jacobsen, N.G., Fuhrman, D.R., Fredsoe, J., 2012. A wave generation toolbox for the open-source CFD library: OpenFoam (R). INTERNATIONAL JOURNAL FOR NUMERICAL METHODS IN FLUIDS 70, 1073-1088. doi 10.1002/fld.2726

Jacobsen, N.G., van Gent, M.R., Wolters, G., 2015. Numerical analysis of the interaction of irregular waves with two dimensional permeable coastal structures. Coastal Engineering 102, 13-29. URL: http://linkinghub.elsevier.com/retrieve/pii/ S0378383915000836 doi $10.1016 / j$.coastaleng.2015.05.004

Jensen, B., Jacobsen, N.G., Christensen, E.D., 2014. Investigations on the porous media equations and resistance coefficients for coastal structures. Coastal Engineering 84, 56-72. URL: http://linkinghub.elsevier.com/retrieve/pii/ S0378383913001816 doi $10.1016 / \mathrm{j}$. coastaleng.2013.11.004

Kristiansen, T., Faltinsen, O.M., 2012. Modelling of current loads on aquaculture net cages. Journal of Fluids and Structures 34, 218-235. URL: http://linkinghub.elsevier.com/retrieve/pii/S0889974612000783, doi 10.1016/j.jfluidstructs. 2012.04.001

Kristiansen, T., Faltinsen, O.M., 2015. Experimental and numerical study of an aquaculture net cage with floater in waves and current. Journal of Fluids and Structures 54, 1-26. URL: http://linkinghub.elsevier.com/retrieve/pii/ S0889974614002114 doi $10.1016 / \mathrm{j} \cdot \mathrm{jfluidstructs.2014.08.015}$

Lader, P.F., Enerhaug, B., Fredheim, A., Krokstad, J., 2003. Modelling of 3D net structures exposed to waves and current, in: Proceedings of International Conference on hydroelasticity in marine technology, pp. 19-26.

Lader, P.F., Fredheim, A., 2006. Dynamic properties of a flexible net sheet in waves and current-A numerical approach. Aquacultural Engineering 35, 228-238. URL: http://linkinghub.elsevier.com/retrieve/pii/S0144860906000161, doi 10. $1016 / j$. aquaeng. 2006.02 .002

Le Tallec, P., Mouro, J., 2001. Fluid structure interaction with large structural displacements. Computer Methods in Applied Mechanics and Engineering 190, 3039-3067. URL: http://linkinghub.elsevier.com/retrieve/pii/S0045782500003819. doi $10.1016 /$ S0045-7825(00) 00381-9

Lessen, M., 1949. On stability of free laminar boundary layer between parallel streams. Technical Report. NASA. United States.

Li, Y.C., Zhao, Y.P., Gui, F.K., Teng, B., 2006. Numerical simulation of the hydrodynamic behaviour of submerged plane nets in current. Ocean Engineering 33, 2352-2368. URL: http://linkinghub.elsevier.com/retrieve/pii/S0029801806000370. doi $10.1016 / j$. oceaneng. 2005.11 .013

Løland, G., 1993. Current forces on, and water flow through and around, floating fish farms. Aquaculture International 1, 72-89. doi $10.1007 / \mathrm{BF} 00692665$

Michler, C., Hulshoff, S.J., van Brummelen, E.H., de Borst, R., 2004. A monolithic approach to fluid-structure interaction. Computers and Fluids 33, 839-848. doi 10.1016/j.compfluid.2003.06.006

Mittal, R., Iaccarino, G., 2005. Immersed Boundary Methods. Annual Review of Fluid Mechanics 37, 239-261. doi 10.1146/ annurev.fluid.37.061903.175743

Moe, H., Fredheim, a., Hopperstad, O.S., 2010. Structural analysis of aquaculture net cages in current. Journal of Fluids and Structures 26, 503-516. URL: http://linkinghub.elsevier.com/retrieve/pii/S0889974610000289, doi 10.1016/j. jfluidstructs.2010.01.007.

Patankar, S., Spalding, D., 1972. A calculation procedure for heat, mass and momentum transfer in three-dimensional parabolic 
flows. International Journal of Heat and Mass Transfer 15, 1787 - 1806. URL: http://www.sciencedirect.com/science/ article/pii/0017931072900543, doi http://dx.doi.org/10.1016/0017-9310(72)90054-3

Patursson, Ø., Swift, M.R., Tsukrov, I., Simonsen, K., Baldwin, K., Fredriksson, D.W., Celikkol, B., 2010. Development of a porous media model with application to flow through and around a net panel. Ocean Engineering 37, 314-324. URL: http://linkinghub.elsevier.com/retrieve/pii/S0029801809002406 doi 10.1016/j.oceaneng.2009.10.001

Rudi, H., land G., L., Furunes, I., 1988. Experiments with nets; forces on and flow through net panels and cage systems. Technical Report MT51 F88-0215. MARINTEK. Trondheim, Norway.

Schlichting, H., Gersten, K., 2000. Boundary Layer Theory. Springer-Verlag Berlin Heidelberg.

Ting, L., 1959. On the mixing of two parallel streams. Journal of Mathematics and Physics 38, 153-165. URL: http: //dx.doi.org/10.1002/sapm1959381153, doi 10.1002/sapm1959381153

Tsukrov, I., Eroshkin, O., Fredriksson, D., Swift, M.R., Celikkol, B., 2002. Finite element modeling of net panels using a consistent net element. Ocean Engineering 30, 251-270.

Zhao, Y.P., Bi, C.W., Dong, G.H., Gui, F.K., Cui, Y., Guan, C.T., Xu, T.J., 2013a. Numerical simulation of the flow around fishing plane nets using the porous media model. Ocean Engineering 62, 25-37. URL: http://linkinghub.elsevier.com/ retrieve/pii/S0029801813000243 doi 10.1016/j.oceaneng.2013.01.009

Zhao, Y.P., Bi, C.W., Dong, G.H., Gui, F.K., Cui, Y., Xu, T.J., 2013b. Numerical simulation of the flow field inside and around gravity cages. Aquacultural Engineering 52, 1-13. URL: http://linkinghub.elsevier.com/retrieve/pii/ S0144860912000593 doi $10.1016 / j$.aquaeng.2012.06.001

Zhao, Y.P., Li, Y.C., Dong, G.H., Gui, F.K., Wu, H., 2008. An experimental and numerical study of hydrodynamic characteristics of submerged flexible plane nets in waves. AQUACULTURAL ENGINEERING 38, 16-25. doi $10.1016 / \mathrm{j}$. aquaeng. 2007.10 .004 